\title{
Polynomial Time Solution of Travelling Salesman Problem
}

\section{Samuel Gheverghese*}

Computer Science and Engineering, TKM College of Engineering, Alleppey District, Kerala, India

\begin{abstract}
This note is for finding a polynomial time solution for the "Travelling Salesman Problem" for a "Complete" graph for any number of vertices. The solutions that I am getting for the examples given in the textbooks are giving the correct answers. If new examples are tried to further test the algorithm and the answers are approximately very near to the correct answer, then this algorithm can be considered as a new type of heuristic algorithm.
\end{abstract}

Keywords: Travelling; Salesman; Problem; Solution; Algorithm

\section{A Brief Description about the Notations}

\section{Beginning of Notations}

A notation is followed by '::-' characters. After '::-' characters, an explanation is given about the notation starting in the next line. In order to make it simple, I have given the examples in the notation itself, in order to explain the notation $[1,2]$.

The vertices of a graph can be alphabets, numbers or a combination of both i.e:- alphanumeric characters.

$$
\text { [] ::- }
$$

The square brackets denote a partial circuit. A partial circuit is a graph in which any vertex of the graph is joined to another vertex by only a single edge. The partial circuit may be empty or it may consists of vertices or both vertices and edges.

\section{[a] ::-}

Denotes a partial circuit containing a single vertex or it denotes a single vertex. Here it denotes a partial circuit containing a vertex named 'a'.

\section{$[\mathrm{a}, \mathrm{b}]::-$}

Denotes that vertex ' $a$ ' is joined to vertex ' $b$ ' or that there is an edge which joins vertex ' $a$ ' with vertex ' $b$ '. Here each vertex is separated by a comma.

\section{$[\mathrm{a}, \mathrm{b}, \mathrm{c}]::-$}

Denotes that vertex ' $a$ ' is joined to vertex ' $b$ ' and vertex ' $b$ ' is joined to vertex 'c'.

So in the '[]' notation, any number of vertices (each vertex separtated by comma) can be written.

\section{$[\mathrm{a}, \mathrm{b}, \mathrm{c}, \mathrm{a}]::-$}

Denotes a partial circuit in which it is also a hamilton circuit, because here vertex ' $a$ ' is joined to vertex ' $b$ ' and vertex ' $b$ ' is joined to vertex ' $c$ ' and vertex 'c' is joined to vertex ' $a$ '. This partial circuit can also be written as $[b, c, a, b]$. So $[a, b, c, a]$ is the same partial circuit as that of $[\mathrm{b}, \mathrm{c}, \mathrm{a}, \mathrm{b}]$.

\section{$\mathrm{w}[\mathrm{a}, \mathrm{b}, \mathrm{c}]::-$}

Denotes the weight of the partial circuit, this weight is the sum total of all the individual weights of the edges of the partial circuit. For eg:- if $\mathrm{w}[\mathrm{a}, \mathrm{b}]=20$ and $\mathrm{w}[\mathrm{b}, \mathrm{c}]=30$, then $\mathrm{w}[\mathrm{a}, \mathrm{b}, \mathrm{c}]=\mathrm{w}[\mathrm{a}, \mathrm{b}]+\mathrm{w}[\mathrm{b}, \mathrm{c}]=20+30=50$.

\section{$[a, b, c]+[b, c, d]=[a, b, c, d]::-$}

Here the two partial circuits $[\mathrm{a}, \mathrm{b}, \mathrm{c}]$ and $[\mathrm{b}, \mathrm{c}, \mathrm{d}]$ are combined to get $[a, b, c, d]$ because the edge of $[b, c]$ is common in both partial circuits. Here the total weight of [a, b, $c, d]$ should be got by taking into consideration the common edge of $[b, c]$. So $w[b, c]$ only needs to be added once.

$$
\begin{aligned}
& \text { For eg:- } \\
& \text { if } \\
& w[a, b, c]=w[a, b]+w[b, c]=20+30=50 \\
& \text { and } \\
& w[b, c, d]=w[b, c]+w[c, d]=30+40=70 \\
& \text { and } \\
& \text { if }[a, b, c, d]=[a, b, c]+[b, c, d] \\
& \text { Then, } \\
& w[a, b, c, d]=w[a, b]+w[b, c]+w[c, d]=20+30+40=90
\end{aligned}
$$

So $w[a, b, c, d]$ is not got by directly adding the weights of $w[a, b, c]$ and $w[b, c, d]$, since there is a common edge of $[b, c]$ present in both $[a, b, c]$ and $[b, c, d]$. So $w[a, b, c, d]$ is not equal to $w[a, b, c]+w[b, c, d]$ ie:not equal to $120(50+70=120)$.

\section{ec[a] ::-}

Denotes an edge count' of the vertex 'a'. An 'edge count' is the number of edges attached to a vertex. For eg:- in $[a, b, c], e c[a]=1$, $\mathrm{ec}[\mathrm{b}]=2$ and $\mathrm{ec}[\mathrm{c}]=1$. Here the edge count of $[\mathrm{b}]$ is 2 since there are 2 edges attached to vertex ' $b$ '. ec[a]=1, because there is only one edge attached to vertex 'a'. In a partial circuit, the edge count of each vertex should be less than or equal to 2, to satisfy the criterion of a Hamilton circuit, when the partial circuit is used to form the Hamilton circuit in the end of the process.

${ }^{*}$ Corresponding author: Samuel Gheverghese, Computer Science and Engineering, TKM College of Engineering, Alleppey District, Kerala, India, Tel: 0474271 3126; E-mail: purplesky1111@yahoo.com

Received April 15, 2015; Accepted May 26, 2015; Published June 02, 2015

Citation: Gheverghese G (2015) Polynomial Time Solution of Travelling Salesman Problem. J Appl Computat Math 4: 219. doi:10.4172/2168-9679.1000219

Copyright: (c) 2015 Gheverghese G. This is an open-access article distributed under the terms of the Creative Commons Attribution License, which permits unrestricted use, distribution, and reproduction in any medium, provided the original author and source are credited. 


\section{Section \#1.m (BEGIN) ::-}

This denotes the section of "Example 1". 'm' denotes any number greater than or equal to one. Also 'BEGIN' denotes the beginning of the section.

\section{Section \#1.m (END) ::-}

This denotes the section of "Example 1". 'm' denotes any number greater than or equal to one. Also 'END' denotes the end of the section.

Section \#2.m (BEGIN) ::-

This denotes the section of "Example 2". 'm' denotes any number greater than or equal to one. Also 'BEGIN' denotes the beginning of the section.

\section{Section \#2.m (END) ::-}

This denotes the section of "Example 2". 'm' denotes any number greater than or equal to one. Also 'END' denotes the end of the section.

$->\{\mathrm{Dm}\}::-$

Here 'D' means description. ' $m$ ' stands for any number greater than or equal to one. This marker ie:- '-->\{Dm\}' gives the statement number which marks a statement which precedes the '-->\{D1\}' marker. This marker is written elsewhere which gives a description of the statement which it marks. This marker is used mainly inside sections when space is required for writing descriptions.

$->$ Dm. ::-

Here ' $\mathrm{D}$ ' means description and ' $m$ ' stands for any number greater than or equal to one. This marker ie:- '->Dm' gives a description of the statement which it has marked.

\section{$\mathrm{C}(\mathrm{n}, \mathrm{r})::-$}

This means a 'combination' of ' $n$ ' number of edges taken ' $r$ ' at a time. Here ' $n$ ' and ' $r$ ' are numbers. This 'combination' is the same combination that is there in mathematics. Here $C(n, r)=n ! /(r ! *(n-r) !)$

End of Notations

\section{Examples}

Below are given some examples for describing properties of joining of partial circuits.

a) Two partial circuits are $[a, b, c]$ and $[b, d, c]$. When these two partial circuits are joined, $[b, c]$ of $[a, b, c]$ can be replaced by $[b, d, c]$. Since one more vertex'd' is added, the edge count of each vertex is not violated (ie:- the edge count of each vertex is less than or equal to 2 ). So the joined partial circuit becomes $[a, b, d, c]$ ( Here the edge $[b, c]$ is replaced by $[\mathrm{b}, \mathrm{d}, \mathrm{c}])$.

b) Two partial circuits are $[a, b, c]$ and $[a, d, c]$. If the Hamilton circuit required to be found consists of only 4 vertices, then these partial circuits can be joined. So if the hamilton circuit consists of only 4 vertices, the the two partial circuits can be joined to get the partial circuit $[\mathrm{a}, \mathrm{b}, \mathrm{c}, \mathrm{d}, \mathrm{a}]$. This partial circuit ie:- $[\mathrm{a}, \mathrm{b}, \mathrm{c}, \mathrm{d}, \mathrm{a}]$ is a hamilton circuit consisting of 4 vertices.

But suppose, if the hamilton circuit required consisted of for eg:- 5 vertices, then these two partial circuits cannot be joined, because if they are joined, then they will already form a hamilton circuit of 4 vertices, which is not what is wanted, but instead a hamilton circuit of 5 vertices is required. Also $[a, b, c]$ cannot be replaced by $[a, d, c]$, because if a new vertex is obtained, in its place another vertex becomes lost, for eg:- if vertex ' $d$ ' is obtained, then vertex ' $b$ ' is lost if $[a, b, c]$ is replaced by [a,d,c].

c) Two partial circuits are $[\mathrm{a}, \mathrm{b}, \mathrm{c}]$ and $[\mathrm{c}, \mathrm{d}, \mathrm{e}]$. These two partial circuits can be joined at the common vertex ' $c$ '. So the partial circuit after joining is [a,b,c,d,e].

d) Two partial circuits are $[a, b, c]$ and $[b, d, e]$. These two partial circuits cannot be joined at the common vertex ' $b$ ', since if the two partial circuits are joined, it would violate the edge count of vertex ' $b$ ' ie:- the edge count of vertex ' $b$ ' would be 3 after joining the partial circuits.

e) Two partial circuits are $[a, b, c]$ and $[c, b, d]$. These two partial circuits cannot be joined at the common edge [b,c], because if the two partial circuits are joined, it would violate the edge count of vertex ' $b$ ' ie:- the edge count of vertex ' $b$ ' would be 3 after joining the partial circuits.

\section{Example 1}

This example is taken from the Book "Elements of Discrete Mathematics" by C.L.Liu. The solution for the example is got in the process described from Section \#1.1 to Section \#1.9.

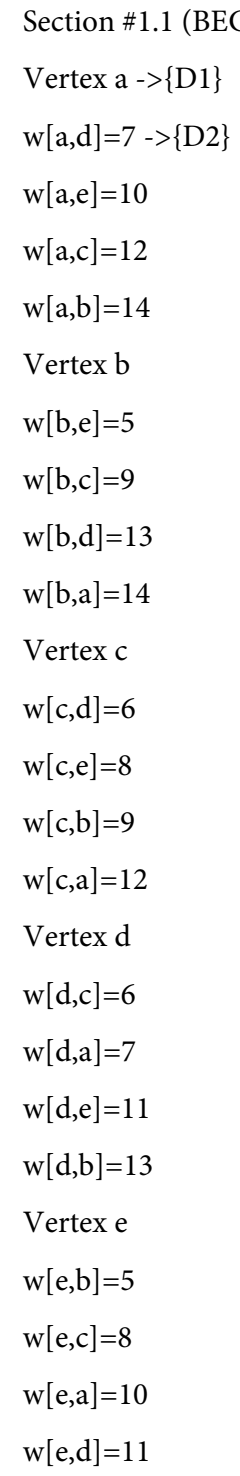


$->$ D1. This shows the heading marked 'Vertex a'.

$->$ D2. This shows the weight of one of the edges (namely edge $[\mathrm{a}, \mathrm{d}]$ ) which is connected to Vertex a.

In this section, the weights of the different edges connected to the different vertices of a 5 vertex graph are shown. The 5 vertex graph is the example taken from the book "Elements of discrete mathematics".

Section \#1.1 (END)

\section{Section \#1.2 (BEGIN)}

In this section a combination of the edges ( the edges are taken from Section \#1.1 ) connected to a particular vertex is taken. For eg:- there are four edges connected to vertex a. So the number of combinations of the four edges connected to 'vertex a' by taking two edges at a time is $\mathrm{C}(4,2)=4 ! /((4-2) ! * 2 !)=6$.

\section{Vertex a}

$$
\begin{aligned}
& {[\mathrm{a}, \mathrm{d}]+[\mathrm{a}, \mathrm{e}]=[\mathrm{d}, \mathrm{a}, \mathrm{e}]} \\
& {[\mathrm{a}, \mathrm{d}]+[\mathrm{a}, \mathrm{c}]=[\mathrm{d}, \mathrm{a}, \mathrm{c}]} \\
& {[\mathrm{a}, \mathrm{d}]+[\mathrm{a}, \mathrm{b}]=[\mathrm{d}, \mathrm{a}, \mathrm{b}]} \\
& {[\mathrm{a}, \mathrm{e}]+[\mathrm{a}, \mathrm{c}]=[\mathrm{e}, \mathrm{a}, \mathrm{c}]} \\
& {[\mathrm{a}, \mathrm{e}]+[\mathrm{a}, \mathrm{b}]=[\mathrm{e}, \mathrm{a}, \mathrm{b}]} \\
& {[\mathrm{a}, \mathrm{c}]+[\mathrm{a}, \mathrm{b}]=[\mathrm{c}, \mathrm{a}, \mathrm{b}]}
\end{aligned}
$$

\section{Vertex b}

$$
\begin{aligned}
& {[\mathrm{b}, \mathrm{e}]+[\mathrm{b}, \mathrm{c}]=[\mathrm{e}, \mathrm{b}, \mathrm{c}]} \\
& {[\mathrm{b}, \mathrm{e}]+[\mathrm{b}, \mathrm{d}]=[\mathrm{e}, \mathrm{b}, \mathrm{d}]} \\
& {[\mathrm{b}, \mathrm{e}]+[\mathrm{b}, \mathrm{a}]=[\mathrm{e}, \mathrm{b}, \mathrm{a}]} \\
& {[\mathrm{b}, \mathrm{c}]+[\mathrm{b}, \mathrm{d}]=[\mathrm{c}, \mathrm{b}, \mathrm{d}]} \\
& {[\mathrm{b}, \mathrm{c}]+[\mathrm{b}, \mathrm{a}]=[\mathrm{c}, \mathrm{b}, \mathrm{a}]} \\
& {[\mathrm{b}, \mathrm{d}]+[\mathrm{b}, \mathrm{a}]=[\mathrm{d}, \mathrm{b}, \mathrm{a}]} \\
& \text { Vertex c } \\
& {[\mathrm{c}, \mathrm{d}]+[\mathrm{c}, \mathrm{e}]=[\mathrm{d}, \mathrm{c}, \mathrm{e}]} \\
& {[\mathrm{c}, \mathrm{d}]+[\mathrm{c}, \mathrm{b}]=[\mathrm{d}, \mathrm{c}, \mathrm{b}]} \\
& {[\mathrm{c}, \mathrm{d}]+[\mathrm{c}, \mathrm{a}]=[\mathrm{d}, \mathrm{c}, \mathrm{a}]} \\
& {[\mathrm{c}, \mathrm{e}]+[\mathrm{c}, \mathrm{b}]=[\mathrm{e}, \mathrm{c}, \mathrm{b}]} \\
& {[\mathrm{c}, \mathrm{e}]+[\mathrm{c}, \mathrm{a}]=[\mathrm{e}, \mathrm{c}, \mathrm{a}]} \\
& {[\mathrm{c}, \mathrm{b}]+[\mathrm{c}, \mathrm{a}]=[\mathrm{b}, \mathrm{c}, \mathrm{a}]}
\end{aligned}
$$

\section{Vertex d}

$$
\begin{aligned}
& {[\mathrm{d}, \mathrm{c}]+[\mathrm{d}, \mathrm{a}]=[\mathrm{c}, \mathrm{d}, \mathrm{a}]} \\
& {[\mathrm{d}, \mathrm{c}]+[\mathrm{d}, \mathrm{e}]=[\mathrm{c}, \mathrm{d}, \mathrm{e}]} \\
& {[\mathrm{d}, \mathrm{c}]+[\mathrm{d}, \mathrm{b}]=[\mathrm{c}, \mathrm{d}, \mathrm{b}]} \\
& {[\mathrm{d}, \mathrm{a}]+[\mathrm{d}, \mathrm{e}]=[\mathrm{a}, \mathrm{d}, \mathrm{e}]} \\
& {[\mathrm{d}, \mathrm{a}]+[\mathrm{d}, \mathrm{b}]=[\mathrm{a}, \mathrm{d}, \mathrm{b}]} \\
& {[\mathrm{d}, \mathrm{e}]+[\mathrm{d}, \mathrm{b}]=[\mathrm{e}, \mathrm{d}, \mathrm{b}]}
\end{aligned}
$$

Vertex e

$$
\begin{aligned}
& {[e, b]+[e, c]=[b, e, c]} \\
& {[e, b]+[e, a]=[b, e, a]} \\
& {[e, b]+[e, d]=[b, e, d]} \\
& {[e, c]+[e, a]=[c, e, a]} \\
& {[e, c]+[e, d]=[c, e, d]} \\
& {[e, a]+[e, d]=[b, e, c]}
\end{aligned}
$$

Section \#1.2 (END)

Section \#1.3 (BEGIN)

In this section, the weights of the partial circuits (these partial circuits were obtained in Section \#1.2 ) of a particular vertex are given below.

$$
\begin{aligned}
& \text { Vertex a } \\
& \mathrm{w}[\mathrm{d}, \mathrm{a}, \mathrm{e}]=7+10=17 \\
& \mathrm{w}[\mathrm{d}, \mathrm{a}, \mathrm{c}]=7+12=19 \\
& \mathrm{w}[\mathrm{d}, \mathrm{a}, \mathrm{b}]=7+14=21 \\
& \mathrm{w}[\mathrm{e}, \mathrm{a}, \mathrm{c}]=10+12=22 \\
& \mathrm{w}[\mathrm{e}, \mathrm{a}, \mathrm{b}]=10+14=24 \\
& \mathrm{w}[\mathrm{c}, \mathrm{a}, \mathrm{b}]=12+14=26 \\
& \mathrm{Vertex} \mathrm{b} \\
& \mathrm{w}[\mathrm{e}, \mathrm{b}, \mathrm{c}]=5+9=14 \\
& \mathrm{w}[\mathrm{e}, \mathrm{b}, \mathrm{d}]=5+13=18 \\
& \mathrm{w}[\mathrm{e}, \mathrm{b}, \mathrm{a}]=5+14=19 \\
& \mathrm{w}[\mathrm{c}, \mathrm{b}, \mathrm{d}]=9+13=22 \\
& \mathrm{w}[\mathrm{c}, \mathrm{b}, \mathrm{a}]=9+14=23 \\
& \mathrm{w}[\mathrm{d}, \mathrm{b}, \mathrm{a}]=13+14=27
\end{aligned}
$$

\section{Vertex c}

$\mathrm{w}[\mathrm{d}, \mathrm{c}, \mathrm{e}]=6+8=14$

$\mathrm{w}[\mathrm{d}, \mathrm{c}, \mathrm{b}]=6+9=15$

$\mathrm{w}[\mathrm{d}, \mathrm{c}, \mathrm{a}]=6+12=18$

$\mathrm{w}[\mathrm{e}, \mathrm{c}, \mathrm{b}]=8+9=17$

$\mathrm{w}[\mathrm{e}, \mathrm{c}, \mathrm{a}]=8+12=20$

$\mathrm{w}[\mathrm{b}, \mathrm{c}, \mathrm{a}]=9+12=21$

Vertex d

$$
\begin{aligned}
& w[c, d, a]=6+7=13 \\
& w[c, d, e]=6+11=17 \\
& w[c, d, b]=6+13=19 \\
& w[a, d, e]=7+11=18 \\
& w[a, d, b]=7+13=20 \\
& w[e, d, b]=11+13=24
\end{aligned}
$$

\section{Vertex e}

$\mathrm{w}[\mathrm{b}, \mathrm{e}, \mathrm{c}]=5+8=13$ 
$\mathrm{w}[\mathrm{b}, \mathrm{e}, \mathrm{a}]=5+10=15$

$\mathrm{w}[\mathrm{b}, \mathrm{e}, \mathrm{d}]=5+11=16$

$\mathrm{w}[\mathrm{c}, \mathrm{e}, \mathrm{a}]=8+10=18$

$\mathrm{w}[\mathrm{c}, \mathrm{e}, \mathrm{d}]=8+11=19$

$\mathrm{w}[\mathrm{b}, \mathrm{e}, \mathrm{c}]=10+11=21$

Section \#1.3 (END)

\section{Section \#1.4 (BEGIN)}

In this section, the partial circuits under each vertex are sorted in 'Ascending order' according to their weights.

Vertex a (sorted)

$$
\begin{aligned}
& {[\mathrm{a}, \mathrm{d}]+[\mathrm{a}, \mathrm{e}]=[\mathrm{d}, \mathrm{a}, \mathrm{e}]} \\
& {[\mathrm{a}, \mathrm{d}]+[\mathrm{a}, \mathrm{c}]=[\mathrm{d}, \mathrm{a}, \mathrm{c}]} \\
& {[\mathrm{a}, \mathrm{d}]+[\mathrm{a}, \mathrm{b}]=[\mathrm{d}, \mathrm{a}, \mathrm{b}]} \\
& {[\mathrm{a}, \mathrm{e}]+[\mathrm{a}, \mathrm{c}]=[\mathrm{e}, \mathrm{a}, \mathrm{c}]} \\
& {[\mathrm{a}, \mathrm{e}]+[\mathrm{a}, \mathrm{b}]=[\mathrm{e}, \mathrm{a}, \mathrm{b}]} \\
& {[\mathrm{a}, \mathrm{c}]+[\mathrm{a}, \mathrm{b}]=[\mathrm{c}, \mathrm{a}, \mathrm{b}]} \\
& \operatorname{Vertex} \mathrm{b}(\mathrm{sorted}) \\
& {[\mathrm{b}, \mathrm{e}]+[\mathrm{b}, \mathrm{c}]=[\mathrm{e}, \mathrm{b}, \mathrm{c}]} \\
& {[\mathrm{b}, \mathrm{e}]+[\mathrm{b}, \mathrm{d}]=[\mathrm{e}, \mathrm{b}, \mathrm{d}]} \\
& {[\mathrm{b}, \mathrm{e}]+[\mathrm{b}, \mathrm{a}]=[\mathrm{e}, \mathrm{b}, \mathrm{a}]} \\
& {[\mathrm{b}, \mathrm{c}]+[\mathrm{b}, \mathrm{d}]=[\mathrm{c}, \mathrm{b}, \mathrm{d}]} \\
& {[\mathrm{b}, \mathrm{c}]+[\mathrm{b}, \mathrm{a}]=[\mathrm{c}, \mathrm{b}, \mathrm{a}]} \\
& {[\mathrm{b}, \mathrm{d}]+[\mathrm{b}, \mathrm{a}]=[\mathrm{d}, \mathrm{b}, \mathrm{a}]}
\end{aligned}
$$

Vertex c (sorted)

$$
\begin{aligned}
& {[\mathrm{c}, \mathrm{d}]+[\mathrm{c}, \mathrm{e}]=[\mathrm{d}, \mathrm{c}, \mathrm{e}]} \\
& {[\mathrm{c}, \mathrm{d}]+[\mathrm{c}, \mathrm{b}]=[\mathrm{d}, \mathrm{c}, \mathrm{b}]} \\
& {[\mathrm{c}, \mathrm{e}]+[\mathrm{c}, \mathrm{b}]=[\mathrm{e}, \mathrm{c}, \mathrm{b}]} \\
& {[\mathrm{c}, \mathrm{d}]+[\mathrm{c}, \mathrm{a}]=[\mathrm{d}, \mathrm{c}, \mathrm{a}]} \\
& {[\mathrm{c}, \mathrm{e}]+[\mathrm{c}, \mathrm{a}]=[\mathrm{e}, \mathrm{c}, \mathrm{a}]} \\
& {[\mathrm{c}, \mathrm{b}]+[\mathrm{c}, \mathrm{a}]=[\mathrm{b}, \mathrm{c}, \mathrm{a}]}
\end{aligned}
$$

\section{Vertex d ( sorted )}

$$
\begin{aligned}
& {[\mathrm{d}, \mathrm{c}]+[\mathrm{d}, \mathrm{a}]=[\mathrm{c}, \mathrm{d}, \mathrm{a}]} \\
& {[\mathrm{d}, \mathrm{c}]+[\mathrm{d}, \mathrm{e}]=[\mathrm{c}, \mathrm{d}, \mathrm{e}]} \\
& {[\mathrm{d}, \mathrm{a}]+[\mathrm{d}, \mathrm{e}]=[\mathrm{a}, \mathrm{d}, \mathrm{e}]} \\
& {[\mathrm{d}, \mathrm{c}]+[\mathrm{d}, \mathrm{b}]=[\mathrm{c}, \mathrm{d}, \mathrm{b}]} \\
& {[\mathrm{d}, \mathrm{a}]+[\mathrm{d}, \mathrm{b}]=[\mathrm{a}, \mathrm{d}, \mathrm{b}]} \\
& {[\mathrm{d}, \mathrm{e}]+[\mathrm{d}, \mathrm{b}]=[\mathrm{e}, \mathrm{d}, \mathrm{b}]}
\end{aligned}
$$$$
\text { Vertex e (sorted) }
$$

$$
\begin{aligned}
& w[d, a, e]=7+10=17 \\
& w[d, a, c]=7+12=19 \\
& w[d, a, b]=7+14=21 \\
& w[e, a, c]=10+12=22 \\
& w[e, a, b]=10+14=24 \\
& w[c, a, b]=12+14=26 \\
& w[e, b, c]=5+9=14 \\
& w[e, b, d]=5+13=18 \\
& w[e, b, a]=5+14=19 \\
& w[c, b, d]=9+13=22 \\
& w[c, b, a]=9+14=23 \\
& w[d, b, a]=13+14=27
\end{aligned}
$$

$\mathrm{w}[\mathrm{d}, \mathrm{c}, \mathrm{e}]=6+8=14$

$\mathrm{w}[\mathrm{d}, \mathrm{c}, \mathrm{b}]=6+9=15$

$\mathrm{w}[\mathrm{e}, \mathrm{c}, \mathrm{b}]=8+9=17$

$\mathrm{w}[\mathrm{d}, \mathrm{c}, \mathrm{a}]=6+12=18$

$\mathrm{w}[\mathrm{e}, \mathrm{c}, \mathrm{a}]=8+12=20$

$\mathrm{w}[\mathrm{b}, \mathrm{c}, \mathrm{a}]=9+12=21$

$\mathrm{w}[\mathrm{c}, \mathrm{d}, \mathrm{a}]=6+7=13$

$\mathrm{w}[\mathrm{c}, \mathrm{d}, \mathrm{e}]=6+11=17$

$\mathrm{w}[\mathrm{a}, \mathrm{d}, \mathrm{e}]=7+11=18$

$\mathrm{w}[\mathrm{c}, \mathrm{d}, \mathrm{b}]=6+13=19$

$\mathrm{w}[\mathrm{a}, \mathrm{d}, \mathrm{b}]=7+13=20$ $\mathrm{w}[\mathrm{e}, \mathrm{d}, \mathrm{b}]=11+13=24$

$$
\begin{array}{ll}
{[e, b]+[e, c]=[b, e, c]} & w[b, e, c]=5+8=13 \\
{[e, b]+[e, a]=[b, e, a]} & w[b, e, a]=5+10=15 \\
{[e, b]+[e, d]=[b, e, d]} & w[b, e, d]=5+11=16 \\
{[e, c]+[e, a]=[c, e, a]} & w[c, e, a]=8+10=18 \\
{[e, c]+[e, d]=[c, e, d]} & w[c, e, d]=8+11=19 \\
{[e, a]+[e, d]=[b, e, c]} & w[b, e, c]=10+11=21
\end{array}
$$

Section \#1.4 (END)

Section \#1.5 (BEGIN)

In this section, the partial circuit with the lowest weight under each vertex is taken individually and written below.

$$
\begin{array}{ll}
{[\mathrm{a}, \mathrm{d}]+[\mathrm{a}, \mathrm{e}]=[\mathrm{d}, \mathrm{a}, \mathrm{e}]} & \mathrm{w}[\mathrm{d}, \mathrm{a}, \mathrm{e}]=7+10=17->\{\mathrm{D} 3\} \\
{[\mathrm{b}, \mathrm{e}]+[\mathrm{b}, \mathrm{c}]=[\mathrm{e}, \mathrm{b}, \mathrm{c}]} & \mathrm{w}[\mathrm{e}, \mathrm{b}, \mathrm{c}]=5+9=14->\{\mathrm{D} 4\} \\
{[\mathrm{c}, \mathrm{d}]+[\mathrm{c}, \mathrm{e}]=[\mathrm{d}, \mathrm{c}, \mathrm{e}]} & \mathrm{w}[\mathrm{d}, \mathrm{c}, \mathrm{e}]=6+8=14 \\
{[\mathrm{~d}, \mathrm{c}]+[\mathrm{d}, \mathrm{a}]=[\mathrm{c}, \mathrm{d}, \mathrm{a}]} & \mathrm{w}[\mathrm{c}, \mathrm{d}, \mathrm{a}]=6+7=13 \\
{[\mathrm{e}, \mathrm{b}]+[\mathrm{e}, \mathrm{c}]=[\mathrm{b}, \mathrm{e}, \mathrm{c}]} & \mathrm{w}[\mathrm{b}, \mathrm{e}, \mathrm{c}]=5+8=13
\end{array}
$$

$->$ D3. Here $[\mathrm{d}, \mathrm{a}, \mathrm{e}]$ is the lowest weight combination taken from Vertex a.

$->\mathrm{D} 4$. Here $[\mathrm{e}, \mathrm{b}, \mathrm{c}]$ is the lowest weight combination taken from Vertex b.

\section{Section \#1.5 (END}

\section{Section \#1.6 (BEGIN)}

In this section, the partial circuits written in Section \#1.5 are sorted in 'Descending order' of their weights and written here.
$[\mathrm{a}, \mathrm{d}]+[\mathrm{a}, \mathrm{e}]=[\mathrm{d}, \mathrm{a}, \mathrm{e}]$
$w[d, a, e]=7+10=17($ vertexa $)->\{D 5\}$
$[\mathrm{b}, \mathrm{e}]+[\mathrm{b}, \mathrm{c}]=[\mathrm{e}, \mathrm{b}, \mathrm{c}]$
$\mathrm{w}[\mathrm{e}, \mathrm{b}, \mathrm{c}]=5+9=14 \quad($ vertex $\mathrm{b})$
$[\mathrm{c}, \mathrm{d}]+[\mathrm{c}, \mathrm{e}]=[\mathrm{d}, \mathrm{c}, \mathrm{e}]$ $\mathrm{w}[\mathrm{d}, \mathrm{c}, \mathrm{e}]=6+8=14 \quad($ vertex $\mathrm{c})$
$[\mathrm{d}, \mathrm{c}]+[\mathrm{d}, \mathrm{a}]=[\mathrm{c}, \mathrm{d}, \mathrm{a}]$
$\mathrm{w}[\mathrm{c}, \mathrm{d}, \mathrm{a}]=6+7=13 \quad($ vertex $\mathrm{d})$
$[e, b]+[e, c]=[b, e, c]$ $\mathrm{w}[\mathrm{b}, \mathrm{e}, \mathrm{c}]=5+8=13 \quad($ vertex e)

$->$ D5 $[\mathrm{d}, \mathrm{a}, \mathrm{e}]$ is a partial circuit having the highest weight in the group in this section.

\section{Section \#1.6 (END)}

Section \#1.7 (BEGIN)

In this section, in order to choose a partial circuit (or the order of which vertex to choose from) is given by the order in Section \#1.6. So the order is as follows ie:- vertex $a$, vertex $b$, vertex $c$, vertex $d$, vertex e.

Vertex a (sorted)

$$
\begin{aligned}
& {[\mathrm{a}, \mathrm{d}]+[\mathrm{a}, \mathrm{e}]=[\mathrm{d}, \mathrm{a}, \mathrm{e}]} \\
& {[\mathrm{a}, \mathrm{d}]+[\mathrm{a}, \mathrm{c}]=[\mathrm{d}, \mathrm{a}, \mathrm{c}]} \\
& {[\mathrm{a}, \mathrm{d}]+[\mathrm{a}, \mathrm{b}]=[\mathrm{d}, \mathrm{a}, \mathrm{b}]} \\
& {[\mathrm{a}, \mathrm{e}]+[\mathrm{a}, \mathrm{c}]=[\mathrm{e}, \mathrm{a}, \mathrm{c}]} \\
& {[\mathrm{a}, \mathrm{e}]+[\mathrm{a}, \mathrm{b}]=[\mathrm{e}, \mathrm{a}, \mathrm{b}]} \\
& {[\mathrm{a}, \mathrm{c}]+[\mathrm{a}, \mathrm{b}]=[\mathrm{c}, \mathrm{a}, \mathrm{b}]}
\end{aligned}
$$

$\mathrm{w}[\mathrm{d}, \mathrm{a}, \mathrm{e}]=7+10=17$

$\mathrm{w}[\mathrm{d}, \mathrm{a}, \mathrm{c}]=7+12=19$

$\mathrm{w}[\mathrm{d}, \mathrm{a}, \mathrm{b}]=7+14=21$

$\mathrm{w}[\mathrm{e}, \mathrm{a}, \mathrm{c}]=10+12=22$

$\mathrm{w}[\mathrm{e}, \mathrm{a}, \mathrm{b}]=10+14=24$

$\mathrm{w}[\mathrm{c}, \mathrm{a}, \mathrm{b}]=12+14=26$ 
Vertex b (sorted)

$$
\begin{aligned}
& {[\mathrm{b}, \mathrm{e}]+[\mathrm{b}, \mathrm{c}]=[\mathrm{e}, \mathrm{b}, \mathrm{c}]} \\
& {[\mathrm{b}, \mathrm{e}]+[\mathrm{b}, \mathrm{d}]=[\mathrm{e}, \mathrm{b}, \mathrm{d}]} \\
& {[\mathrm{b}, \mathrm{e}]+[\mathrm{b}, \mathrm{a}]=[\mathrm{e}, \mathrm{b}, \mathrm{a}]} \\
& {[\mathrm{b}, \mathrm{c}]+[\mathrm{b}, \mathrm{d}]=[\mathrm{c}, \mathrm{b}, \mathrm{d}]} \\
& {[\mathrm{b}, \mathrm{c}]+[\mathrm{b}, \mathrm{a}]=[\mathrm{c}, \mathrm{b}, \mathrm{a}]} \\
& {[\mathrm{b}, \mathrm{d}]+[\mathrm{b}, \mathrm{a}]=[\mathrm{d}, \mathrm{b}, \mathrm{a}]} \\
& \text { Vertex c (sorted) }
\end{aligned}
$$$$
[\mathrm{c}, \mathrm{d}]+[\mathrm{c}, \mathrm{e}]=[\mathrm{d}, \mathrm{c}, \mathrm{e}]
$$$$
[c, d]+[c, b]=[d, c, b]
$$$$
[c, e]+[c, b]=[e, c, b]
$$$$
[\mathrm{c}, \mathrm{d}]+[\mathrm{c}, \mathrm{a}]=[\mathrm{d}, \mathrm{c}, \mathrm{a}]
$$$$
[c, e]+[c, a]=[e, c, a]
$$$$
[\mathrm{c}, \mathrm{b}]+[\mathrm{c}, \mathrm{a}]=[\mathrm{b}, \mathrm{c}, \mathrm{a}]
$$$$
\text { Vertex d (sorted) }
$$$$
[\mathrm{d}, \mathrm{c}]+[\mathrm{d}, \mathrm{a}]=[\mathrm{c}, \mathrm{d}, \mathrm{a}]
$$$$
[\mathrm{d}, \mathrm{c}]+[\mathrm{d}, \mathrm{e}]=[\mathrm{c}, \mathrm{d}, \mathrm{e}]
$$$$
[\mathrm{d}, \mathrm{a}]+[\mathrm{d}, \mathrm{e}]=[\mathrm{a}, \mathrm{d}, \mathrm{e}]
$$$$
[\mathrm{d}, \mathrm{c}]+[\mathrm{d}, \mathrm{b}]=[\mathrm{c}, \mathrm{d}, \mathrm{b}]
$$$$
[\mathrm{d}, \mathrm{a}]+[\mathrm{d}, \mathrm{b}]=[\mathrm{a}, \mathrm{d}, \mathrm{b}]
$$$$
[\mathrm{d}, \mathrm{e}]+[\mathrm{d}, \mathrm{b}]=[\mathrm{e}, \mathrm{d}, \mathrm{b}]
$$$$
\text { Vertex e (sorted) }
$$$$
[e, b]+[e, c]=[b, e, c]
$$$$
[e, b]+[e, a]=[b, e, a]
$$$$
[e, b]+[e, d]=[b, e, d]
$$$$
[e, c]+[e, a]=[c, e, a]
$$$$
[e, c]+[e, d]=[c, e, d]
$$$$
[e, a]+[e, d]=[b, e, c]
$$

$\mathrm{w}[\mathrm{e}, \mathrm{b}, \mathrm{c}]=5+9=14$

$\mathrm{w}[\mathrm{e}, \mathrm{b}, \mathrm{d}]=5+13=18$

$\mathrm{w}[\mathrm{e}, \mathrm{b}, \mathrm{a}]=5+14=19$

$\mathrm{w}[\mathrm{c}, \mathrm{b}, \mathrm{d}]=9+13=22$

$\mathrm{w}[\mathrm{c}, \mathrm{b}, \mathrm{a}]=9+14=23$

$\mathrm{w}[\mathrm{d}, \mathrm{b}, \mathrm{a}]=13+14=27$

$\mathrm{w}[\mathrm{d}, \mathrm{c}, \mathrm{e}]=6+8=14$

$\mathrm{w}[\mathrm{d}, \mathrm{c}, \mathrm{b}]=6+9=15$

$\mathrm{w}[\mathrm{e}, \mathrm{c}, \mathrm{b}]=8+9=17$

$\mathrm{w}[\mathrm{d}, \mathrm{c}, \mathrm{a}]=6+12=18$

$\mathrm{w}[\mathrm{e}, \mathrm{c}, \mathrm{a}]=8+12=20$

$\mathrm{w}[\mathrm{b}, \mathrm{c}, \mathrm{a}]=9+12=21$

$\mathrm{w}[\mathrm{c}, \mathrm{d}, \mathrm{a}]=6+7=13$

$\mathrm{w}[\mathrm{c}, \mathrm{d}, \mathrm{e}]=6+11=17$

$\mathrm{w}[\mathrm{a}, \mathrm{d}, \mathrm{e}]=7+11=18$

$\mathrm{w}[\mathrm{c}, \mathrm{d}, \mathrm{b}]=6+13=19$

$\mathrm{w}[\mathrm{a}, \mathrm{d}, \mathrm{b}]=7+13=20$

$\mathrm{w}[\mathrm{e}, \mathrm{d}, \mathrm{b}]=11+13=24$

$\mathrm{w}[\mathrm{b}, \mathrm{e}, \mathrm{c}]=5+8=13$

$\mathrm{w}[\mathrm{b}, \mathrm{e}, \mathrm{a}]=5+10=15$

$\mathrm{w}[\mathrm{b}, \mathrm{e}, \mathrm{d}]=5+11=16$

$\mathrm{w}[\mathrm{c}, \mathrm{e}, \mathrm{a}]=8+10=18$

$\mathrm{w}[\mathrm{c}, \mathrm{e}, \mathrm{d}]=8+11=19$

$\mathrm{w}[\mathrm{b}, \mathrm{e}, \mathrm{c}]=10+11=21$

Section \#1.7 (END)

\section{Section \#1.8 (BEGIN)}

Now from Section \#1.7, take a partial circuit from each vertex (the order of the vertex to be taken, should be the same order in which the vertices are written in Section \#1.7) and join them together in order to form a hamilton circuit. The process is as follows:-

1) Take $[d, a, e]$ from Vertex a. Since hamilton circuit rule is not violated, $[\mathrm{d}, \mathrm{a}, \mathrm{e}]$ is taken.

2) Take $[e, b, c]$ from Vertex b. $[e, b, c]$ fits with $[d, a, e]$. So $[e, b, c]$ is also taken. The new partial circuit is $[\mathrm{d}, \mathrm{a}, \mathrm{e}, \mathrm{b}, \mathrm{c}]$.

3) Take $[d, c, e]$ from Vertex c. $[d, c, e]$ does not fit with $[d, a, e, b, c]$. So the next step is not to go to the next vertex but to take another partial circuit from the same vertex to see if it fits with $[d, a, e, b, c]$. So take $[\mathrm{d}, \mathrm{c}, \mathrm{b}]$ from Vertex c. [d,c,b] fits with [d,a,e,b,c]. Fitting [d,c,b] with $[\mathrm{d}, \mathrm{a}, \mathrm{e}, \mathrm{b}, \mathrm{c}]$ creates the hamilton circuit $[\mathrm{d}, \mathrm{a}, \mathrm{e}, \mathrm{b}, \mathrm{c}, \mathrm{d}]$. The weight of the hamilton circuit $w[d, a, e, b, c, d]=w[d, a]+w[a, e]+w[e, b]+w[b, c]+$ $\mathrm{w}[\mathrm{c}, \mathrm{d}]=7+10+5+9+6=37$.

$$
\text { Also }[\mathrm{d}, \mathrm{a}, \mathrm{e}, \mathrm{b}, \mathrm{c}, \mathrm{d}]=[\mathrm{d}, \mathrm{a}, \mathrm{e}]+[\mathrm{e}, \mathrm{b}, \mathrm{c}]+[\mathrm{d}, \mathrm{c}, \mathrm{b}] \text {. }
$$

The Hamilton circuit was obtained, and to know if it is the minimum circuit, checking is needed if any lower partial circuits which have been bypassed will form lesser weight Hamilton circuits. To know which all are the lesser weight partial circuits, the next section ( ie:Section \#1.9 ) onwards shows the lesser weight partial circuits.

\section{Section \#1.8 (END)}

Section \#1.9 (BEGIN)

Group the partial circuits of the different vertices present in Section \#1.7 into one group and then sort it. So first grouping the partial circuits, we obtain as below:-

$$
\begin{aligned}
& {[\mathrm{a}, \mathrm{d}]+[\mathrm{a}, \mathrm{e}]=[\mathrm{d}, \mathrm{a}, \mathrm{e}]} \\
& {[a, d]+[a, c]=[d, a, c]} \\
& {[\mathrm{a}, \mathrm{d}]+[\mathrm{a}, \mathrm{b}]=[\mathrm{d}, \mathrm{a}, \mathrm{b}]} \\
& {[\mathrm{a}, \mathrm{e}]+[\mathrm{a}, \mathrm{c}]=[\mathrm{e}, \mathrm{a}, \mathrm{c}]} \\
& {[\mathrm{a}, \mathrm{e}]+[\mathrm{a}, \mathrm{b}]=[\mathrm{e}, \mathrm{a}, \mathrm{b}]} \\
& {[\mathrm{a}, \mathrm{c}]+[\mathrm{a}, \mathrm{b}]=[\mathrm{c}, \mathrm{a}, \mathrm{b}]} \\
& {[b, e]+[b, c]=[e, b, c]} \\
& {[\mathrm{b}, \mathrm{e}]+[\mathrm{b}, \mathrm{d}]=[\mathrm{e}, \mathrm{b}, \mathrm{d}]} \\
& {[\mathrm{b}, \mathrm{e}]+[\mathrm{b}, \mathrm{a}]=[\mathrm{e}, \mathrm{b}, \mathrm{a}]} \\
& {[\mathrm{b}, \mathrm{c}]+[\mathrm{b}, \mathrm{d}]=[\mathrm{c}, \mathrm{b}, \mathrm{d}]} \\
& {[\mathrm{b}, \mathrm{c}]+[\mathrm{b}, \mathrm{a}]=[\mathrm{c}, \mathrm{b}, \mathrm{a}]} \\
& {[\mathrm{b}, \mathrm{d}]+[\mathrm{b}, \mathrm{a}]=[\mathrm{d}, \mathrm{b}, \mathrm{a}]} \\
& {[\mathrm{c}, \mathrm{d}]+[\mathrm{c}, \mathrm{e}]=[\mathrm{d}, \mathrm{c}, \mathrm{e}]} \\
& {[\mathrm{c}, \mathrm{d}]+[\mathrm{c}, \mathrm{b}]=[\mathrm{d}, \mathrm{c}, \mathrm{b}]} \\
& {[c, e]+[c, b]=[e, c, b]} \\
& {[\mathrm{c}, \mathrm{d}]+[\mathrm{c}, \mathrm{a}]=[\mathrm{d}, \mathrm{c}, \mathrm{a}]} \\
& {[\mathrm{c}, \mathrm{e}]+[\mathrm{c}, \mathrm{a}]=[\mathrm{e}, \mathrm{c}, \mathrm{a}]} \\
& {[\mathrm{c}, \mathrm{b}]+[\mathrm{c}, \mathrm{a}]=[\mathrm{b}, \mathrm{c}, \mathrm{a}]} \\
& {[\mathrm{d}, \mathrm{c}]+[\mathrm{d}, \mathrm{a}]=[\mathrm{c}, \mathrm{d}, \mathrm{a}]} \\
& {[\mathrm{d}, \mathrm{c}]+[\mathrm{d}, \mathrm{e}]=[\mathrm{c}, \mathrm{d}, \mathrm{e}]} \\
& {[\mathrm{d}, \mathrm{a}]+[\mathrm{d}, \mathrm{e}]=[\mathrm{a}, \mathrm{d}, \mathrm{e}]} \\
& {[\mathrm{d}, \mathrm{c}]+[\mathrm{d}, \mathrm{b}]=[\mathrm{c}, \mathrm{d}, \mathrm{b}]} \\
& {[\mathrm{d}, \mathrm{a}]+[\mathrm{d}, \mathrm{b}]=[\mathrm{a}, \mathrm{d}, \mathrm{b}]} \\
& {[\mathrm{d}, \mathrm{e}]+[\mathrm{d}, \mathrm{b}]=[\mathrm{e}, \mathrm{d}, \mathrm{b}]} \\
& {[e, b]+[e, c]=[b, e, c]} \\
& {[e, b]+[e, a]=[b, e, a]} \\
& {[e, b]+[e, d]=[b, e, d]} \\
& {[e, c]+[e, a]=[c, e, a]} \\
& {[e, c]+[e, d]=[c, e, d]} \\
& {[e, a]+[e, d]=[b, e, c]}
\end{aligned}
$$

$\mathrm{w}[\mathrm{d}, \mathrm{a}, \mathrm{e}]=7+10=17$

$\mathrm{w}[\mathrm{d}, \mathrm{a}, \mathrm{c}]=7+12=19$

$\mathrm{w}[\mathrm{d}, \mathrm{a}, \mathrm{b}]=7+14=21$

$\mathrm{w}[\mathrm{e}, \mathrm{a}, \mathrm{c}]=10+12=22$

$\mathrm{w}[\mathrm{e}, \mathrm{a}, \mathrm{b}]=10+14=24$

$\mathrm{w}[\mathrm{c}, \mathrm{a}, \mathrm{b}]=12+14=26$

$\mathrm{w}[\mathrm{e}, \mathrm{b}, \mathrm{c}]=5+9=14$

$\mathrm{w}[\mathrm{e}, \mathrm{b}, \mathrm{d}]=5+13=18$

$\mathrm{w}[\mathrm{e}, \mathrm{b}, \mathrm{a}]=5+14=19$

$\mathrm{w}[\mathrm{c}, \mathrm{b}, \mathrm{d}]=9+13=22$

$\mathrm{w}[\mathrm{c}, \mathrm{b}, \mathrm{a}]=9+14=23$

$\mathrm{w}[\mathrm{d}, \mathrm{b}, \mathrm{a}]=13+14=27$

$\mathrm{w}[\mathrm{d}, \mathrm{c}, \mathrm{e}]=6+8=14$

$\mathrm{w}[\mathrm{d}, \mathrm{c}, \mathrm{b}]=6+9=15$

$\mathrm{w}[\mathrm{e}, \mathrm{c}, \mathrm{b}]=8+9=17$

$\mathrm{w}[\mathrm{d}, \mathrm{c}, \mathrm{a}]=6+12=18$

$\mathrm{w}[\mathrm{e}, \mathrm{c}, \mathrm{a}]=8+12=20$

$\mathrm{w}[\mathrm{b}, \mathrm{c}, \mathrm{a}]=9+12=21$

$\mathrm{w}[\mathrm{c}, \mathrm{d}, \mathrm{a}]=6+7=13$

$\mathrm{w}[\mathrm{c}, \mathrm{d}, \mathrm{e}]=6+11=17$

$\mathrm{w}[\mathrm{a}, \mathrm{d}, \mathrm{e}]=7+11=18$

$\mathrm{w}[\mathrm{c}, \mathrm{d}, \mathrm{b}]=6+13=19$

$\mathrm{w}[\mathrm{a}, \mathrm{d}, \mathrm{b}]=7+13=20$

$\mathrm{w}[\mathrm{e}, \mathrm{d}, \mathrm{b}]=11+13=24$

$\mathrm{w}[\mathrm{b}, \mathrm{e}, \mathrm{c}]=5+8=13$

$\mathrm{w}[\mathrm{b}, \mathrm{e}, \mathrm{a}]=5+10=15$

$\mathrm{w}[\mathrm{b}, \mathrm{e}, \mathrm{d}]=5+11=16$

$\mathrm{w}[\mathrm{c}, \mathrm{e}, \mathrm{a}]=8+10=18$

$\mathrm{w}[\mathrm{c}, \mathrm{e}, \mathrm{d}]=8+11=19$

$\mathrm{w}[\mathrm{b}, \mathrm{e}, \mathrm{c}]=10+11=21$ 


\section{Section \#1.9 (END)}

\section{Section \#1.10 (BEGIN)}

Now sort the group obtained in Section \#1.9 according to their weights.

The sorted lists of the different partial circuits are as follows.

$$
\begin{aligned}
& {[\mathrm{d}, \mathrm{c}]+[\mathrm{d}, \mathrm{a}]=[\mathrm{c}, \mathrm{d}, \mathrm{a}] \quad \mathrm{w}[\mathrm{c}, \mathrm{d}, \mathrm{a}]=6+7=13} \\
& {[e, b]+[e, c]=[b, e, c]} \\
& {[\mathrm{b}, \mathrm{e}]+[\mathrm{b}, \mathrm{c}]=[\mathrm{e}, \mathrm{b}, \mathrm{c}]} \\
& {[\mathrm{c}, \mathrm{d}]+[\mathrm{c}, \mathrm{e}]=[\mathrm{d}, \mathrm{c}, \mathrm{e}]} \\
& {[\mathrm{c}, \mathrm{d}]+[\mathrm{c}, \mathrm{b}]=[\mathrm{d}, \mathrm{c}, \mathrm{b}]} \\
& {[e, b]+[e, a]=[b, e, a]} \\
& {[\mathrm{e}, \mathrm{b}]+[\mathrm{e}, \mathrm{d}]=[\mathrm{b}, \mathrm{e}, \mathrm{d}]} \\
& {[\mathrm{a}, \mathrm{d}]+[\mathrm{a}, \mathrm{e}]=[\mathrm{d}, \mathrm{a}, \mathrm{e}]} \\
& {[c, e]+[c, b]=[e, c, b]} \\
& {[\mathrm{d}, \mathrm{c}]+[\mathrm{d}, \mathrm{e}]=[\mathrm{c}, \mathrm{d}, \mathrm{e}]} \\
& {[\mathrm{b}, \mathrm{e}]+[\mathrm{b}, \mathrm{d}]=[\mathrm{e}, \mathrm{b}, \mathrm{d}]} \\
& {[\mathrm{c}, \mathrm{d}]+[\mathrm{c}, \mathrm{a}]=[\mathrm{d}, \mathrm{c}, \mathrm{a}]} \\
& {[\mathrm{d}, \mathrm{a}]+[\mathrm{d}, \mathrm{e}]=[\mathrm{a}, \mathrm{d}, \mathrm{e}]} \\
& {[e, c]+[e, a]=[c, e, a]} \\
& {[\mathrm{a}, \mathrm{d}]+[\mathrm{a}, \mathrm{c}]=[\mathrm{d}, \mathrm{a}, \mathrm{c}]} \\
& {[\mathrm{b}, \mathrm{e}]+[\mathrm{b}, \mathrm{a}]=[\mathrm{e}, \mathrm{b}, \mathrm{a}]} \\
& {[\mathrm{d}, \mathrm{c}]+[\mathrm{d}, \mathrm{b}]=[\mathrm{c}, \mathrm{d}, \mathrm{b}]} \\
& {[e, c]+[e, d]=[c, e, d]} \\
& {[c, e]+[c, a]=[e, c, a]} \\
& {[\mathrm{d}, \mathrm{a}]+[\mathrm{d}, \mathrm{b}]=[\mathrm{a}, \mathrm{d}, \mathrm{b}]} \\
& {[\mathrm{a}, \mathrm{d}]+[\mathrm{a}, \mathrm{b}]=[\mathrm{d}, \mathrm{a}, \mathrm{b}]} \\
& {[\mathrm{c}, \mathrm{b}]+[\mathrm{c}, \mathrm{a}]=[\mathrm{b}, \mathrm{c}, \mathrm{a}]} \\
& {[e, a]+[e, d]=[b, e, c]} \\
& {[\mathrm{a}, \mathrm{e}]+[\mathrm{a}, \mathrm{c}]=[\mathrm{e}, \mathrm{a}, \mathrm{c}]} \\
& {[\mathrm{b}, \mathrm{c}]+[\mathrm{b}, \mathrm{d}]=[\mathrm{c}, \mathrm{b}, \mathrm{d}]} \\
& {[\mathrm{b}, \mathrm{c}]+[\mathrm{b}, \mathrm{a}]=[\mathrm{c}, \mathrm{b}, \mathrm{a}]} \\
& {[a, e]+[a, b]=[e, a, b]} \\
& {[\mathrm{d}, \mathrm{e}]+[\mathrm{d}, \mathrm{b}]=[\mathrm{e}, \mathrm{d}, \mathrm{b}]} \\
& {[a, c]+[a, b]=[c, a, b]} \\
& {[\mathrm{b}, \mathrm{d}]+[\mathrm{b}, \mathrm{a}]=[\mathrm{d}, \mathrm{b}, \mathrm{a}]} \\
& \begin{array}{l}
w[\mathrm{c}, \mathrm{d}, \mathrm{a}]=6+7=13 \\
\mathrm{w}[\mathrm{b}, \mathrm{e}, \mathrm{c}]=5+8=13
\end{array} \\
& w[e, b, c]=5+9=14->\{D 7\} \\
& \mathrm{w}[\mathrm{d}, \mathrm{c}, \mathrm{e}]=6+8=14 \\
& \mathrm{w}[\mathrm{d}, \mathrm{c}, \mathrm{b}]=6+9=15->\{\mathrm{D} 8\} \\
& \mathrm{w}[\mathrm{b}, \mathrm{e}, \mathrm{a}]=5+10=15 \\
& \mathrm{w}[\mathrm{b}, \mathrm{e}, \mathrm{d}]=5+11=16 \\
& \mathrm{w}[\mathrm{d}, \mathrm{a}, \mathrm{e}]=7+10=17->\{\mathrm{D} 6\} \\
& \mathrm{w}[\mathrm{e}, \mathrm{c}, \mathrm{b}]=8+9=17 \\
& \mathrm{w}[\mathrm{c}, \mathrm{d}, \mathrm{e}]=6+11=17 \\
& \mathrm{w}[\mathrm{e}, \mathrm{b}, \mathrm{d}]=5+13=18 \\
& \mathrm{w}[\mathrm{d}, \mathrm{c}, \mathrm{a}]=6+12=18 \\
& \mathrm{w}[\mathrm{a}, \mathrm{d}, \mathrm{e}]=7+11=18 \\
& \mathrm{w}[\mathrm{c}, \mathrm{e}, \mathrm{a}]=8+10=18 \\
& \mathrm{w}[\mathrm{d}, \mathrm{a}, \mathrm{c}]=7+12=19 \\
& \mathrm{w}[\mathrm{e}, \mathrm{b}, \mathrm{a}]=5+14=19 \\
& \mathrm{w}[\mathrm{c}, \mathrm{d}, \mathrm{b}]=6+13=19 \\
& \mathrm{w}[\mathrm{c}, \mathrm{e}, \mathrm{d}]=8+11=19 \\
& \mathrm{w}[\mathrm{e}, \mathrm{c}, \mathrm{a}]=8+12=20 \\
& \mathrm{w}[\mathrm{a}, \mathrm{d}, \mathrm{b}]=7+13=20 \\
& \mathrm{w}[\mathrm{d}, \mathrm{a}, \mathrm{b}]=7+14=21 \\
& \mathrm{w}[\mathrm{b}, \mathrm{c}, \mathrm{a}]=9+12=21 \\
& \mathrm{w}[\mathrm{b}, \mathrm{e}, \mathrm{c}]=10+11=21 \\
& \mathrm{w}[\mathrm{e}, \mathrm{a}, \mathrm{c}]=10+12=22 \\
& \mathrm{w}[\mathrm{c}, \mathrm{b}, \mathrm{d}]=9+13=22 \\
& \mathrm{w}[\mathrm{c}, \mathrm{b}, \mathrm{a}]=9+14=23 \\
& \mathrm{w}[\mathrm{e}, \mathrm{a}, \mathrm{b}]=10+14=24 \\
& \mathrm{w}[\mathrm{e}, \mathrm{d}, \mathrm{b}]=11+13=24 \\
& \mathrm{w}[\mathrm{c}, \mathrm{a}, \mathrm{b}]=12+14=26 \\
& \mathrm{w}[\mathrm{d}, \mathrm{b}, \mathrm{a}]=13+14=27
\end{aligned}
$$

$[c, d, a],[b, e, c],[d, c, e],[b, e, a]$ and $[b, e, d]$ was not used in forming the hamilton circuits even though they had lesser weights. So these partial circuits are taken individually and are used to form hamilton circuits and to find whether these hamilton circuits have lesser weight than the hamilton circuit found out earlier (ie:- $[\mathrm{d}, \mathrm{a}, \mathrm{e}, \mathrm{b}, \mathrm{c}, \mathrm{d}]$ was the hamilton circuit found out earlier). (The lesser weight partial circuits which were not used while forming the hamilton circuits for [c,d,a], $[b, e, c],[d, c, e],[b, e, a]$ and $[b, e, d]$ need not be used for processing again).

Now first $[\mathrm{c}, \mathrm{d}, \mathrm{a}]$ has to be taken as a necessary partial circuit and then the same process that occurred in Section \#1.8 has to be done. 'Necessary partial circuit' means that $[\mathrm{c}, \mathrm{d}, \mathrm{a}]$ should be used as the first partial circuit for finding the hamilton circuit.

1) So first $[c, d, a]$ is taken. Since hamilton circuit rule is not violated, $[\mathrm{c}, \mathrm{d}, \mathrm{a}]$ is taken.

2) Take $[d, a, e]$ from Vertex a. $[d, a, e]$ fits with $[c, d, a]$. So $[d, a, e]$ is taken. The new partial circuit is $[\mathrm{c}, \mathrm{d}, \mathrm{a}, \mathrm{e}]$.

3) Take $[e, b, c]$ from Vertex b. $[e, b, c]$ fits with $[c, d, a, e]$. So $[e, b, c]$ is taken. The new hamilton circuit formed is $[\mathrm{c}, \mathrm{d}, \mathrm{a}, \mathrm{e}, \mathrm{b}, \mathrm{c}]$. The weight of the hamilton circuit $w[c, d, a, e, b, c]=w[c, d]+w[d, a]+w[a, e]+w[e, b]+$ $\mathrm{w}[\mathrm{b}, \mathrm{c}]=6+7+10+5+9=37$

Therefore $[\mathrm{c}, \mathrm{d}, \mathrm{a}, \mathrm{e}, \mathrm{b}, \mathrm{c}]=[\mathrm{c}, \mathrm{d}, \mathrm{a}]+[\mathrm{d}, \mathrm{a}, \mathrm{e}]+[\mathrm{e}, \mathrm{b}, \mathrm{c}]$.

$\mathrm{w}[\mathrm{c}, \mathrm{d}, \mathrm{a}, \mathrm{e}, \mathrm{b}, \mathrm{c}]=37$.

Now $[\mathrm{b}, \mathrm{e}, \mathrm{c}]$ has to be taken as a necessary partial circuit and then the same process that occurred in Section \#1.8 has to be done.

1) First $[b, e, c]$ is taken. Since hamilton circuit rule is not violated, $[\mathrm{b}, \mathrm{e}, \mathrm{c}]$ is taken.

2) Take $[d, a, e]$ from Vertex a. $[d, a, e]$ does not fit with $[b, e, c]$. So take the next partial circuit from Vertex a. Take $[\mathrm{d}, \mathrm{a}, \mathrm{c}]$ from Vertex a. $[d, a, c]$ fits with $[b, e, c]$. So the new partial circuit is $[b, e, c, a, d]$. Since a partial circuit from Vertex a which fits is got, then a partial circuit from the next vertex is got.

3) Take $[e, b, c]$ from Vertex b. [e,b,c] does not fit with $[b, e, c, a, d]$. So take [e,b,d] from Vertex b. [e,b,d] fits with [b,e,c,a,d]. Fitting [e,b,d] with $[\mathrm{b}, \mathrm{e}, \mathrm{c}, \mathrm{a}, \mathrm{d}]$ creates the hamilton circuit $[\mathrm{b}, \mathrm{e}, \mathrm{c}, \mathrm{a}, \mathrm{d}, \mathrm{b}]$. The weight of the hamilton circuit $w[b, e, c, a, d, b]=w[b, e]+w[e, c]+w[c, a]+w[a, d]+$ $\mathrm{w}[\mathrm{d}, \mathrm{b}]=5+8+12+7+13=45$

Therefore $[\mathrm{b}, \mathrm{e}, \mathrm{c}, \mathrm{a}, \mathrm{d}, \mathrm{b}]=[\mathrm{b}, \mathrm{e}, \mathrm{c}]+[\mathrm{d}, \mathrm{a}, \mathrm{c}]+[\mathrm{e}, \mathrm{b}, \mathrm{d}]$

$\mathrm{w}[\mathrm{b}, \mathrm{e}, \mathrm{c}, \mathrm{a}, \mathrm{d}, \mathrm{b}]=45$.

Now $[\mathrm{d}, \mathrm{c}, \mathrm{e}]$ has to be taken as a necessary partial circuit and then the same process that occurred in Section \#1.8 has to be done.

1) First $[d, c, e]$ is taken. Since hamilton circuit rule is not violated, $[\mathrm{d}, \mathrm{c}, \mathrm{e}]$ is taken.

2) Take $[d, a, e]$ from Vertex a. $[d, a, e]$ does not fit with $[d, c, e]$. So take $[d, a, c]$ from Vertex a. Here $[d, a, c]$ can replace $[d, c]$ of $[d, c, e]$, since according to the properties of joining of partial circuits, it is allowed. So $[d, a, c]$ fits with $[d, c, e]$. So the new partial circuit is $[d, a, c, e]$.

3) Take $[e, b, c]$ from Vertex b. Here $[e, b, c]$ can replace $[c, e]$ of [d,a,c,e], since according to the properties of joining of partial circuits, it is allowed. So $[e, b, c]$ fits with $[d, a, c, e]$. So the new partial circuit is $[\mathrm{d}, \mathrm{a}, \mathrm{c}, \mathrm{b}, \mathrm{e}]$.

4) Take $[d, c, e]$ from Vertex c. [d,c,e] does not fit with $[d, a, c, b, e]$. So also $[d, c, b],[e, c, b],[d, c, a]$ and $[e, c, a]$ from vertex $c$ does not fit with $[d, a, c, b, e] .[b, c, a]$ from vertex $c$ is already present in $[d, a, c, b, e]$. So all partial circuits from Vertex $c$ are exhausted. So go to the next vertex ie:- Vertex d. 
5) Take $[c, d, a]$ from Vertex d. [c,d,a] does not fit with $[d, a, c, b, e]$. Take $[c, d, e]$ from vertex $d$. [c,d,e] does not fit with $[\mathrm{d}, \mathrm{a}, \mathrm{c}, \mathrm{b}, \mathrm{e}]$. Take $[\mathrm{a}, \mathrm{d}, \mathrm{e}]$ from Vertex d. [a,d,e] fits with $[\mathrm{d}, \mathrm{a}, \mathrm{c}, \mathrm{b}, \mathrm{e}]$ to create the hamilton circuit $[\mathrm{d}, \mathrm{a}, \mathrm{c}, \mathrm{b}, \mathrm{e}, \mathrm{d}]$. The weight of the hamilton circuit $\mathrm{w}[\mathrm{d}, \mathrm{a}, \mathrm{c}, \mathrm{b}, \mathrm{e}, \mathrm{d}]=\mathrm{w}[\mathrm{d}, \mathrm{a}]+$ $w[a, c]+w[c, b]+w[b, e]+w[e, d]=44$

$$
\begin{aligned}
& \text { So }[\mathrm{d}, \mathrm{a}, \mathrm{c}, \mathrm{b}, \mathrm{e}, \mathrm{d}]=[\mathrm{d}, \mathrm{c}, \mathrm{e}]+[\mathrm{d}, \mathrm{a}, \mathrm{c}]+[\mathrm{e}, \mathrm{b}, \mathrm{c}]+[\mathrm{a}, \mathrm{d}, \mathrm{e}] \\
& \mathrm{w}[\mathrm{d}, \mathrm{a}, \mathrm{c}, \mathrm{b}, \mathrm{e}, \mathrm{d}]=44 .
\end{aligned}
$$

Now take $[\mathrm{b}, \mathrm{e}, \mathrm{a}]$ as a necessary partial circuit and then the same process that occurred in Section \#1.8 has to be done.

1) First $[b, e, a]$ is taken. Since hamilton circuit rule is not violated, $[\mathrm{b}, \mathrm{e}, \mathrm{a}]$ is taken.

2) Take $[d, a, e]$ from Vertex a. [d,a,e] fits with $[b, e, a]$. So take $[d, a, e]$ from Vertex a. The new partial circuit is $[b, e, a, d]$.

3) Take $[e, b, c]$ from Vertex b. [e,b,c] fits with $[b, e, a, d]$. The new partial circuit is $[\mathrm{c}, \mathrm{b}, \mathrm{e}, \mathrm{a}, \mathrm{d}]$.

4) Take $[d, c, e]$ from Vertex c. [d,c,e] does not fit with $[c, b, e, a, d]$. Take $[\mathrm{d}, \mathrm{c}, \mathrm{b}]$ from Vertex $\mathrm{c}$. [d,c,b] fits with $[\mathrm{c}, \mathrm{b}, \mathrm{e}, \mathrm{a}, \mathrm{d}]$ to form the hamilton circuit $[\mathrm{c}, \mathrm{b}, \mathrm{e}, \mathrm{a}, \mathrm{d}, \mathrm{c}]$.

The hamilton circuit for $[\mathrm{b}, \mathrm{e}, \mathrm{a}]$ is :-

$$
\begin{aligned}
& {[\mathrm{b}, \mathrm{e}, \mathrm{a}]+[\mathrm{d}, \mathrm{a}, \mathrm{e}]+[\mathrm{e}, \mathrm{b}, \mathrm{c}]+[\mathrm{d}, \mathrm{c}, \mathrm{b}]=[\mathrm{b}, \mathrm{e}, \mathrm{a}, \mathrm{d}, \mathrm{c}, \mathrm{b}]} \\
& \mathrm{w}[\mathrm{b}, \mathrm{e}, \mathrm{a}, \mathrm{d}, \mathrm{c}, \mathrm{b}]=37
\end{aligned}
$$

In the same way that the hamilton circuit has been obtained for $[c, d, a],[b, e, c],[d, c, e]$ and $[b, e, a]$, it is also done here for $[b, e, d]$. The result is only written here.

Now taking $[\mathrm{b}, \mathrm{e}, \mathrm{d}]$ as a necessary partial circuit. The hamilton circuit for $[\mathrm{b}, \mathrm{e}, \mathrm{d}]$ is :-

$$
\begin{aligned}
& {[\mathrm{b}, \mathrm{e}, \mathrm{d}]+[\mathrm{d}, \mathrm{a}, \mathrm{e}]+[\mathrm{e}, \mathrm{b}, \mathrm{c}]+[\mathrm{d}, \mathrm{c}, \mathrm{b}]=[\mathrm{b}, \mathrm{e}, \mathrm{a}, \mathrm{d}, \mathrm{c}, \mathrm{b}]} \\
& \mathrm{w}[\mathrm{b}, \mathrm{e}, \mathrm{a}, \mathrm{d}, \mathrm{c}, \mathrm{b}]=37
\end{aligned}
$$

The lowest weight for a hamilton circuit is 37 among the hamilton circuits taken for the partial circuits of $[c, d, a],[b, e, c],[d, c, e],[b, e, a]$ and $[\mathrm{b}, \mathrm{e}, \mathrm{d}]$. Also the lowest weight of the Hamilton circuit obtained in Section \#1.8 is also 37. So the Hamilton circuit obtained in Section \#1.8 or in Section \#1.10 with lowest weight of "37" is taken as the minimum weight Hamilton circuit.

Section \#1.10 (END)

\section{Example 2}

This example is taken from "Section 3.4" of the Book "A First Look at Graph Theory" by John Clark and Derek Allan Holton. The solution for the example is got in the process described from Section \#2.1 to Section \#2.8. "Example 2" is solved in the same way as "Example 1" was solved.

$$
\begin{aligned}
& \text { Vertex b } \\
& w[b, c]=10 \\
& w[b, m]=11 \\
& w[b, r]=11 \\
& w[b, s]=6 \\
& w[b, v]=10
\end{aligned}
$$$$
\text { Section \#2.1 (BEGIN) }
$$

$$
\begin{aligned}
& \text { Vertex v } \\
& \mathrm{w}[\mathrm{v}, \mathrm{b}]=10 \\
& \mathrm{w}[\mathrm{v}, \mathrm{c}]=20 \\
& \mathrm{w}[\mathrm{v}, \mathrm{m}]=19 \\
& \mathrm{w}[\mathrm{v}, \mathrm{r}]=20 \\
& \mathrm{w}[\mathrm{v}, \mathrm{s}]=11 \\
& \text { Vertex s } \\
& \mathrm{w}[\mathrm{s}, \mathrm{v}]=11 \\
& \mathrm{w}[\mathrm{s}, \mathrm{b}]=6 \\
& \mathrm{w}[\mathrm{s}, \mathrm{c}]=15 \\
& \mathrm{w}[\mathrm{s}, \mathrm{m}]=15 \\
& \mathrm{w}[\mathrm{s}, \mathrm{r}]=15
\end{aligned}
$$

In this section, the weights of the different edges connected to the different vertices of a 6 vertex graph are shown.

\section{Section \#2.1 (END)}

\section{Section \#2.2 (BEGIN)}

In this section a combination of the edges connected to a particular vertex is taken. For eg:- there are five edges connected to vertex b. So the number of combinations of the five edges connected to 'vertex b' by taking two edges at a time is $C(5,2)=5 ! /((5-2) ! * 2 !)=10$.

Also the weights of the partial circuits of a particular vertex are also written to the right of the partial circuits.

Vertex b

$[b, c]+[b, m]=[c, b, m]$

$\mathrm{w}[\mathrm{c}, \mathrm{b}, \mathrm{m}]=10+11=21$ 
Citation: Gheverghese G (2015) Polynomial Time Solution of Travelling Salesman Problem. J Appl Computat Math 4: 219. doi:10.4172/21689679.1000219

Page 8 of 14

$$
\begin{aligned}
& {[\mathrm{b}, \mathrm{c}]+[\mathrm{b}, \mathrm{r}]=[\mathrm{c}, \mathrm{b}, \mathrm{r}]} \\
& {[\mathrm{b}, \mathrm{c}]+[\mathrm{b}, \mathrm{s}]=[\mathrm{c}, \mathrm{b}, \mathrm{s}]} \\
& {[\mathrm{b}, \mathrm{c}]+[\mathrm{b}, \mathrm{v}]=[\mathrm{c}, \mathrm{b}, \mathrm{v}]} \\
& {[\mathrm{b}, \mathrm{m}]+[\mathrm{b}, \mathrm{r}]=[\mathrm{m}, \mathrm{b}, \mathrm{r}]} \\
& {[\mathrm{b}, \mathrm{m}]+[\mathrm{b}, \mathrm{s}]=[\mathrm{m}, \mathrm{b}, \mathrm{s}]} \\
& {[\mathrm{b}, \mathrm{m}]+[\mathrm{b}, \mathrm{v}]=[\mathrm{m}, \mathrm{b}, \mathrm{v}]} \\
& {[\mathrm{b}, \mathrm{r}]+[\mathrm{b}, \mathrm{s}]=[\mathrm{r}, \mathrm{b}, \mathrm{s}]} \\
& {[\mathrm{b}, \mathrm{r}]+[\mathrm{b}, \mathrm{v}]=[\mathrm{r}, \mathrm{b}, \mathrm{v}]} \\
& {[\mathrm{b}, \mathrm{s}]+[\mathrm{b}, \mathrm{v}]=[\mathrm{s}, \mathrm{b}, \mathrm{v}]} \\
& \mathrm{Vertex} \mathrm{v} \\
& {[\mathrm{v}, \mathrm{b}]+[\mathrm{v}, \mathrm{c}]=[\mathrm{b}, \mathrm{v}, \mathrm{c}]} \\
& {[\mathrm{v}, \mathrm{b}]+[\mathrm{v}, \mathrm{m}]=[\mathrm{b}, \mathrm{v}, \mathrm{m}]} \\
& {[\mathrm{v}, \mathrm{b}]+[\mathrm{v}, \mathrm{r}]=[\mathrm{b}, \mathrm{v}, \mathrm{r}]} \\
& {[\mathrm{v}, \mathrm{b}]+[\mathrm{v}, \mathrm{s}]=[\mathrm{b}, \mathrm{v}, \mathrm{s}]} \\
& {[\mathrm{v}, \mathrm{c}]+[\mathrm{v}, \mathrm{m}]=[\mathrm{c}, \mathrm{v}, \mathrm{m}]} \\
& {[\mathrm{v}, \mathrm{c}]+[\mathrm{v}, \mathrm{r}]=[\mathrm{c}, \mathrm{v}, \mathrm{r}]} \\
& {[\mathrm{v}, \mathrm{c}]+[\mathrm{v}, \mathrm{s}]=[\mathrm{c}, \mathrm{v}, \mathrm{s}]} \\
& {[\mathrm{v}, \mathrm{m}]+[\mathrm{v}, \mathrm{r}]=[\mathrm{m}, \mathrm{v}, \mathrm{r}]} \\
& {[\mathrm{v}, \mathrm{m}]+[\mathrm{v}, \mathrm{s}]=[\mathrm{m}, \mathrm{v}, \mathrm{s}]} \\
& {[\mathrm{v}, \mathrm{r}]+[\mathrm{v}, \mathrm{s}]=[\mathrm{r}, \mathrm{v}, \mathrm{s}]} \\
&
\end{aligned}
$$

\section{Vertex s}

$$
\begin{aligned}
& {[\mathrm{s}, \mathrm{v}]+[\mathrm{s}, \mathrm{b}]=[\mathrm{v}, \mathrm{s}, \mathrm{b}]} \\
& {[\mathrm{s}, \mathrm{v}]+[\mathrm{s}, \mathrm{c}]=[\mathrm{v}, \mathrm{s}, \mathrm{c}]} \\
& {[\mathrm{s}, \mathrm{v}]+[\mathrm{s}, \mathrm{m}]=[\mathrm{v}, \mathrm{s}, \mathrm{m}]} \\
& {[\mathrm{s}, \mathrm{v}]+[\mathrm{s}, \mathrm{r}]=[\mathrm{v}, \mathrm{s}, \mathrm{r}]} \\
& {[\mathrm{s}, \mathrm{b}]+[\mathrm{s}, \mathrm{c}]=[\mathrm{b}, \mathrm{s}, \mathrm{c}]} \\
& {[\mathrm{s}, \mathrm{b}]+[\mathrm{s}, \mathrm{m}]=[\mathrm{b}, \mathrm{s}, \mathrm{m}]} \\
& {[\mathrm{s}, \mathrm{b}]+[\mathrm{s}, \mathrm{r}]=[\mathrm{b}, \mathrm{s}, \mathrm{r}]} \\
& {[\mathrm{s}, \mathrm{c}]+[\mathrm{s}, \mathrm{m}]=[\mathrm{c}, \mathrm{s}, \mathrm{m}]} \\
& {[\mathrm{s}, \mathrm{c}]+[\mathrm{s}, \mathrm{r}]=[\mathrm{c}, \mathrm{s}, \mathrm{r}]} \\
& {[\mathrm{s}, \mathrm{m}]+[\mathrm{s}, \mathrm{r}]=[\mathrm{m}, \mathrm{s}, \mathrm{r}]} \\
& \mathrm{Vertex} \mathrm{r} \\
& {[\mathrm{r}, \mathrm{s}]+[\mathrm{r}, \mathrm{v}]=[\mathrm{s}, \mathrm{r}, \mathrm{v}]} \\
& {[\mathrm{r}, \mathrm{s}]+[\mathrm{r}, \mathrm{b}]=[\mathrm{s}, \mathrm{r}, \mathrm{b}]} \\
& {[\mathrm{r}, \mathrm{s}]+[\mathrm{r}, \mathrm{c}]=[\mathrm{s}, \mathrm{r}, \mathrm{c}]} \\
& {[\mathrm{r}, \mathrm{s}]+[\mathrm{r}, \mathrm{m}]=[\mathrm{s}, \mathrm{r}, \mathrm{m}]} \\
& {[\mathrm{r}, \mathrm{v}]+[\mathrm{r}, \mathrm{b}]=[\mathrm{v}, \mathrm{r}, \mathrm{b}]} \\
& {[\mathrm{r}, \mathrm{v}]+[\mathrm{r}, \mathrm{c}]=[\mathrm{v}, \mathrm{r}, \mathrm{c}]} \\
& {[\mathrm{r}, \mathrm{v}]+[\mathrm{r}, \mathrm{m}]=[\mathrm{v}, \mathrm{r}, \mathrm{m}]} \\
& {[\mathrm{r}, \mathrm{b}]+[\mathrm{r}, \mathrm{c}]=[\mathrm{b}, \mathrm{r}, \mathrm{c}]}
\end{aligned}
$$

$$
\begin{aligned}
& \mathrm{w}[\mathrm{c}, \mathrm{b}, \mathrm{r}]=10+11=21 \\
& \mathrm{w}[\mathrm{c}, \mathrm{b}, \mathrm{s}]=10+6=16 \\
& \mathrm{w}[\mathrm{c}, \mathrm{b}, \mathrm{v}]=10+10=20 \\
& \mathrm{w}[\mathrm{m}, \mathrm{b}, \mathrm{r}]=11+11=22 \\
& \mathrm{w}[\mathrm{m}, \mathrm{b}, \mathrm{s}]=11+6=17 \\
& \mathrm{w}[\mathrm{m}, \mathrm{b}, \mathrm{v}]=11+10=21 \\
& \mathrm{w}[\mathrm{r}, \mathrm{b}, \mathrm{s}]=11+6=17 \\
& \mathrm{w}[\mathrm{r}, \mathrm{b}, \mathrm{v}]=11+10=21 \\
& \mathrm{w}[\mathrm{s}, \mathrm{b}, \mathrm{v}]=6+10=16
\end{aligned}
$$$$
\mathrm{w}[\mathrm{b}, \mathrm{v}, \mathrm{c}]=10+20=30
$$$$
\mathrm{w}[\mathrm{b}, \mathrm{v}, \mathrm{m}]=10+19=29
$$$$
\mathrm{w}[\mathrm{b}, \mathrm{v}, \mathrm{r}]=10+20=30
$$$$
\mathrm{w}[\mathrm{b}, \mathrm{v}, \mathrm{s}]=10+11=21
$$$$
\mathrm{w}[\mathrm{c}, \mathrm{v}, \mathrm{m}]=20+19=39
$$$$
\mathrm{w}[\mathrm{c}, \mathrm{v}, \mathrm{r}]=20+20=40
$$$$
\mathrm{w}[\mathrm{c}, \mathrm{v}, \mathrm{s}]=20+11=31
$$$$
\mathrm{w}[\mathrm{m}, \mathrm{v}, \mathrm{r}]=19+20=39
$$$$
\mathrm{w}[\mathrm{m}, \mathrm{v}, \mathrm{s}]=19+11=30
$$$$
\mathrm{w}[\mathrm{r}, \mathrm{v}, \mathrm{s}]=20+11=31
$$$$
\mathrm{w}[\mathrm{v}, \mathrm{s}, \mathrm{b}]=11+6=17
$$$$
\mathrm{w}[\mathrm{v}, \mathrm{s}, \mathrm{c}]=11+15=26
$$$$
\mathrm{w}[\mathrm{v}, \mathrm{s}, \mathrm{m}]=11+15=26
$$$$
\mathrm{w}[\mathrm{v}, \mathrm{s}, \mathrm{r}]=11+15=26
$$$$
\mathrm{w}[\mathrm{b}, \mathrm{s}, \mathrm{c}]=6+15=21
$$$$
\mathrm{w}[\mathrm{b}, \mathrm{s}, \mathrm{m}]=6+15=21
$$$$
\mathrm{w}[\mathrm{b}, \mathrm{s}, \mathrm{r}]=6+15=21
$$$$
\mathrm{w}[\mathrm{c}, \mathrm{s}, \mathrm{m}]=15+15=30
$$$$
\mathrm{w}[\mathrm{c}, \mathrm{s}, \mathrm{r}]=15+15=30
$$$$
\mathrm{w}[\mathrm{m}, \mathrm{s}, \mathrm{r}]=15+15=30
$$

$\mathrm{w}[\mathrm{s}, \mathrm{r}, \mathrm{v}]=15+20=35$

$\mathrm{w}[\mathrm{s}, \mathrm{r}, \mathrm{b}]=15+11=26$

$\mathrm{w}[\mathrm{s}, \mathrm{r}, \mathrm{c}]=15+15=30$

$\mathrm{w}[\mathrm{s}, \mathrm{r}, \mathrm{m}]=15+10=25$

$\mathrm{w}[\mathrm{v}, \mathrm{r}, \mathrm{b}]=20+11=31$

$\mathrm{w}[\mathrm{v}, \mathrm{r}, \mathrm{c}]=20+15=35$

$\mathrm{w}[\mathrm{v}, \mathrm{r}, \mathrm{m}]=20+10=30$

$\mathrm{w}[\mathrm{b}, \mathrm{r}, \mathrm{c}]=11+15=26$ $[\mathrm{r}, \mathrm{b}]+[\mathrm{r}, \mathrm{m}]=[\mathrm{b}, \mathrm{r}, \mathrm{m}]$

$[\mathrm{r}, \mathrm{c}]+[\mathrm{r}, \mathrm{m}]=[\mathrm{c}, \mathrm{r}, \mathrm{m}]$

$\mathrm{w}[\mathrm{b}, \mathrm{r}, \mathrm{m}]=11+10=21$

$\mathrm{w}[\mathrm{c}, \mathrm{r}, \mathrm{m}]=15+10=25$

\section{Vertex m}

$[\mathrm{m}, \mathrm{r}]+[\mathrm{m}, \mathrm{s}]=[\mathrm{r}, \mathrm{m}, \mathrm{s}]$

$\mathrm{w}[\mathrm{r}, \mathrm{m}, \mathrm{s}]=10+15=25$

$[\mathrm{m}, \mathrm{r}]+[\mathrm{m}, \mathrm{v}]=[\mathrm{r}, \mathrm{m}, \mathrm{v}]$

$[\mathrm{m}, \mathrm{r}]+[\mathrm{m}, \mathrm{b}]=[\mathrm{r}, \mathrm{m}, \mathrm{b}]$

$[\mathrm{m}, \mathrm{r}]+[\mathrm{m}, \mathrm{c}]=[\mathrm{r}, \mathrm{m}, \mathrm{c}]$

$[\mathrm{m}, \mathrm{s}]+[\mathrm{m}, \mathrm{v}]=[\mathrm{s}, \mathrm{m}, \mathrm{v}]$

$[\mathrm{m}, \mathrm{s}]+[\mathrm{m}, \mathrm{b}]=[\mathrm{s}, \mathrm{m}, \mathrm{b}]$

$[\mathrm{m}, \mathrm{s}]+[\mathrm{m}, \mathrm{c}]=[\mathrm{s}, \mathrm{m}, \mathrm{c}]$

$[\mathrm{m}, \mathrm{v}]+[\mathrm{m}, \mathrm{b}]=[\mathrm{v}, \mathrm{m}, \mathrm{b}]$

$[\mathrm{m}, \mathrm{v}]+[\mathrm{m}, \mathrm{c}]=[\mathrm{v}, \mathrm{m}, \mathrm{c}]$

$[\mathrm{m}, \mathrm{b}]+[\mathrm{m}, \mathrm{c}]=[\mathrm{b}, \mathrm{m}, \mathrm{c}]$

Vertex c

$[\mathrm{c}, \mathrm{m}]+[\mathrm{c}, \mathrm{r}]=[\mathrm{m}, \mathrm{c}, \mathrm{r}]$

$[\mathrm{c}, \mathrm{m}]+[\mathrm{c}, \mathrm{s}]=[\mathrm{m}, \mathrm{c}, \mathrm{s}]$

$[\mathrm{c}, \mathrm{m}]+[\mathrm{c}, \mathrm{v}]=[\mathrm{m}, \mathrm{c}, \mathrm{v}]$

$[\mathrm{c}, \mathrm{m}]+[\mathrm{c}, \mathrm{b}]=[\mathrm{m}, \mathrm{c}, \mathrm{b}]$

$[\mathrm{c}, \mathrm{r}]+[\mathrm{c}, \mathrm{s}]=[\mathrm{r}, \mathrm{c}, \mathrm{s}]$

$[\mathrm{c}, \mathrm{r}]+[\mathrm{c}, \mathrm{v}]=[\mathrm{r}, \mathrm{c}, \mathrm{v}]$

$[\mathrm{c}, \mathrm{r}]+[\mathrm{c}, \mathrm{b}]=[\mathrm{r}, \mathrm{c}, \mathrm{b}]$

$[\mathrm{c}, \mathrm{s}]+[\mathrm{c}, \mathrm{v}]=[\mathrm{s}, \mathrm{c}, \mathrm{v}]$

$[\mathrm{c}, \mathrm{s}]+[\mathrm{c}, \mathrm{b}]=[\mathrm{s}, \mathrm{c}, \mathrm{b}]$

$[\mathrm{c}, \mathrm{v}]+[\mathrm{c}, \mathrm{b}]=[\mathrm{v}, \mathrm{c}, \mathrm{b}]$

$\mathrm{w}[\mathrm{r}, \mathrm{m}, \mathrm{v}]=10+19=29$

$\mathrm{w}[\mathrm{r}, \mathrm{m}, \mathrm{b}]=10+11=21$

$\mathrm{w}[\mathrm{r}, \mathrm{m}, \mathrm{c}]=10+17=27$

$\mathrm{w}[\mathrm{s}, \mathrm{m}, \mathrm{v}]=15+19=34$

$\mathrm{w}[\mathrm{s}, \mathrm{m}, \mathrm{b}]=15+11=26$

$\mathrm{w}[\mathrm{s}, \mathrm{m}, \mathrm{c}]=15+17=32$

$\mathrm{w}[\mathrm{v}, \mathrm{m}, \mathrm{b}]=19+11=30$

$\mathrm{w}[\mathrm{v}, \mathrm{m}, \mathrm{c}]=19+17=36$

$\mathrm{w}[\mathrm{b}, \mathrm{m}, \mathrm{c}]=11+17=28$

$\mathrm{w}[\mathrm{m}, \mathrm{c}, \mathrm{r}]=17+15=32$

$\mathrm{w}[\mathrm{m}, \mathrm{c}, \mathrm{s}]=17+15=32$

$\mathrm{w}[\mathrm{m}, \mathrm{c}, \mathrm{v}]=17+20=37$

$\mathrm{w}[\mathrm{m}, \mathrm{c}, \mathrm{b}]=17+10=27$

$\mathrm{w}[\mathrm{r}, \mathrm{c}, \mathrm{s}]=15+15=30$

$\mathrm{w}[\mathrm{r}, \mathrm{c}, \mathrm{v}]=15+20=35$

$\mathrm{w}[\mathrm{r}, \mathrm{c}, \mathrm{b}]=15+10=25$

$\mathrm{w}[\mathrm{s}, \mathrm{c}, \mathrm{v}]=15+20=35$

$\mathrm{w}[\mathrm{s}, \mathrm{c}, \mathrm{b}]=15+10=25$

$\mathrm{w}[\mathrm{v}, \mathrm{c}, \mathrm{b}]=20+10=30$

Section \#2.2 (END)

Section \#2.3 (BEGIN)

In this section, the partial circuits under each vertex are sorted in 'Ascending order' according to their weights.

Vertex b (sorted)

$[\mathrm{b}, \mathrm{c}]+[\mathrm{b}, \mathrm{s}]=[\mathrm{c}, \mathrm{b}, \mathrm{s}]$

$\mathrm{w}[\mathrm{c}, \mathrm{b}, \mathrm{s}]=10+6=16$

$[\mathrm{b}, \mathrm{s}]+[\mathrm{b}, \mathrm{v}]=[\mathrm{s}, \mathrm{b}, \mathrm{v}]$

$[\mathrm{b}, \mathrm{m}]+[\mathrm{b}, \mathrm{s}]=[\mathrm{m}, \mathrm{b}, \mathrm{s}]$

$\mathrm{w}[\mathrm{s}, \mathrm{b}, \mathrm{v}]=6+10=16$

$\mathrm{w}[\mathrm{m}, \mathrm{b}, \mathrm{s}]=11+6=17$

$[\mathrm{b}, \mathrm{r}]+[\mathrm{b}, \mathrm{s}]=[\mathrm{r}, \mathrm{b}, \mathrm{s}]$

$\mathrm{w}[\mathrm{r}, \mathrm{b}, \mathrm{s}]=11+6=17$

$[\mathrm{b}, \mathrm{c}]+[\mathrm{b}, \mathrm{v}]=[\mathrm{c}, \mathrm{b}, \mathrm{v}]$

$\mathrm{w}[\mathrm{c}, \mathrm{b}, \mathrm{v}]=10+10=20$

$[b, c]+[b, m]=[c, b, m]$

$[\mathrm{b}, \mathrm{c}]+[\mathrm{b}, \mathrm{r}]=[\mathrm{c}, \mathrm{b}, \mathrm{r}]$

$[\mathrm{b}, \mathrm{m}]+[\mathrm{b}, \mathrm{v}]=[\mathrm{m}, \mathrm{b}, \mathrm{v}]$

$[\mathrm{b}, \mathrm{r}]+[\mathrm{b}, \mathrm{v}]=[\mathrm{r}, \mathrm{b}, \mathrm{v}]$

$[\mathrm{b}, \mathrm{m}]+[\mathrm{b}, \mathrm{r}]=[\mathrm{m}, \mathrm{b}, \mathrm{r}]$

$\mathrm{w}[\mathrm{c}, \mathrm{b}, \mathrm{m}]=10+11=21$

$\mathrm{w}[\mathrm{c}, \mathrm{b}, \mathrm{r}]=10+11=21$

$\mathrm{w}[\mathrm{m}, \mathrm{b}, \mathrm{v}]=11+10=21$

$\mathrm{w}[\mathrm{r}, \mathrm{b}, \mathrm{v}]=11+10=21$

$\mathrm{w}[\mathrm{m}, \mathrm{b}, \mathrm{r}]=11+11=22$ 


$$
\begin{aligned}
& {[\mathrm{v}, \mathrm{b}]+[\mathrm{v}, \mathrm{s}]=[\mathrm{b}, \mathrm{v}, \mathrm{s}]} \\
& {[\mathrm{v}, \mathrm{b}]+[\mathrm{v}, \mathrm{m}]=[\mathrm{b}, \mathrm{v}, \mathrm{m}]} \\
& {[\mathrm{v}, \mathrm{b}]+[\mathrm{v}, \mathrm{c}]=[\mathrm{b}, \mathrm{v}, \mathrm{c}]} \\
& {[\mathrm{v}, \mathrm{b}]+[\mathrm{v}, \mathrm{r}]=[\mathrm{b}, \mathrm{v}, \mathrm{r}]} \\
& {[\mathrm{v}, \mathrm{m}]+[\mathrm{v}, \mathrm{s}]=[\mathrm{m}, \mathrm{v}, \mathrm{s}]} \\
& {[\mathrm{v}, \mathrm{c}]+[\mathrm{v}, \mathrm{s}]=[\mathrm{c}, \mathrm{v}, \mathrm{s}]} \\
& {[\mathrm{v}, \mathrm{r}]+[\mathrm{v}, \mathrm{s}]=[\mathrm{r}, \mathrm{v}, \mathrm{s}]} \\
& {[\mathrm{v}, \mathrm{c}]+[\mathrm{v}, \mathrm{m}]=[\mathrm{c}, \mathrm{v}, \mathrm{m}]} \\
& {[\mathrm{v}, \mathrm{m}]+[\mathrm{v}, \mathrm{r}]=[\mathrm{m}, \mathrm{v}, \mathrm{r}]} \\
& {[\mathrm{v}, \mathrm{c}]+[\mathrm{v}, \mathrm{r}]=[\mathrm{c}, \mathrm{v}, \mathrm{r}]}
\end{aligned}
$$$$
\text { Vertex s (sorted) }
$$$$
[\mathrm{s}, \mathrm{v}]+[\mathrm{s}, \mathrm{b}]=[\mathrm{v}, \mathrm{s}, \mathrm{b}]
$$$$
[\mathrm{s}, \mathrm{b}]+[\mathrm{s}, \mathrm{c}]=[\mathrm{b}, \mathrm{s}, \mathrm{c}]
$$$$
[\mathrm{s}, \mathrm{b}]+[\mathrm{s}, \mathrm{m}]=[\mathrm{b}, \mathrm{s}, \mathrm{m}]
$$$$
[\mathrm{s}, \mathrm{b}]+[\mathrm{s}, \mathrm{r}]=[\mathrm{b}, \mathrm{s}, \mathrm{r}]
$$$$
[\mathrm{s}, \mathrm{v}]+[\mathrm{s}, \mathrm{c}]=[\mathrm{v}, \mathrm{s}, \mathrm{c}]
$$$$
[\mathrm{s}, \mathrm{v}]+[\mathrm{s}, \mathrm{m}]=[\mathrm{v}, \mathrm{s}, \mathrm{m}]
$$$$
[\mathrm{s}, \mathrm{v}]+[\mathrm{s}, \mathrm{r}]=[\mathrm{v}, \mathrm{s}, \mathrm{r}]
$$$$
[\mathrm{s}, \mathrm{c}]+[\mathrm{s}, \mathrm{m}]=[\mathrm{c}, \mathrm{s}, \mathrm{m}]
$$$$
[\mathrm{s}, \mathrm{c}]+[\mathrm{s}, \mathrm{r}]=[\mathrm{c}, \mathrm{s}, \mathrm{r}]
$$$$
[\mathrm{s}, \mathrm{m}]+[\mathrm{s}, \mathrm{r}]=[\mathrm{m}, \mathrm{s}, \mathrm{r}]
$$$$
\text { Vertex r (sorted) }
$$$$
[\mathrm{r}, \mathrm{b}]+[\mathrm{r}, \mathrm{m}]=[\mathrm{b}, \mathrm{r}, \mathrm{m}]
$$$$
[\mathrm{r}, \mathrm{s}]+[\mathrm{r}, \mathrm{m}]=[\mathrm{s}, \mathrm{r}, \mathrm{m}]
$$$$
[\mathrm{r}, \mathrm{c}]+[\mathrm{r}, \mathrm{m}]=[\mathrm{c}, \mathrm{r}, \mathrm{m}]
$$$$
[\mathrm{r}, \mathrm{s}]+[\mathrm{r}, \mathrm{b}]=[\mathrm{s}, \mathrm{r}, \mathrm{b}]
$$$$
[\mathrm{r}, \mathrm{b}]+[\mathrm{r}, \mathrm{c}]=[\mathrm{b}, \mathrm{r}, \mathrm{c}]
$$$$
[\mathrm{r}, \mathrm{s}]+[\mathrm{r}, \mathrm{c}]=[\mathrm{s}, \mathrm{r}, \mathrm{c}]
$$$$
[\mathrm{r}, \mathrm{v}]+[\mathrm{r}, \mathrm{m}]=[\mathrm{v}, \mathrm{r}, \mathrm{m}]
$$$$
[\mathrm{r}, \mathrm{v}]+[\mathrm{r}, \mathrm{b}]=[\mathrm{v}, \mathrm{r}, \mathrm{b}]
$$$$
[\mathrm{r}, \mathrm{s}]+[\mathrm{r}, \mathrm{v}]=[\mathrm{s}, \mathrm{r}, \mathrm{v}]
$$$$
[\mathrm{r}, \mathrm{v}]+[\mathrm{r}, \mathrm{c}]=[\mathrm{v}, \mathrm{r}, \mathrm{c}]
$$

Vertex m (sorted)

$[\mathrm{m}, \mathrm{r}]+[\mathrm{m}, \mathrm{b}]=[\mathrm{r}, \mathrm{m}, \mathrm{b}]$

$[\mathrm{m}, \mathrm{r}]+[\mathrm{m}, \mathrm{s}]=[\mathrm{r}, \mathrm{m}, \mathrm{s}]$

$[\mathrm{m}, \mathrm{s}]+[\mathrm{m}, \mathrm{b}]=[\mathrm{s}, \mathrm{m}, \mathrm{b}]$

$[\mathrm{m}, \mathrm{r}]+[\mathrm{m}, \mathrm{c}]=[\mathrm{r}, \mathrm{m}, \mathrm{c}]$

$[\mathrm{m}, \mathrm{b}]+[\mathrm{m}, \mathrm{c}]=[\mathrm{b}, \mathrm{m}, \mathrm{c}]$

$[\mathrm{m}, \mathrm{r}]+[\mathrm{m}, \mathrm{v}]=[\mathrm{r}, \mathrm{m}, \mathrm{v}]$

$[\mathrm{m}, \mathrm{v}]+[\mathrm{m}, \mathrm{b}]=[\mathrm{v}, \mathrm{m}, \mathrm{b}]$ $\mathrm{w}[\mathrm{b}, \mathrm{v}, \mathrm{s}]=10+11=21$

$\mathrm{w}[\mathrm{b}, \mathrm{v}, \mathrm{m}]=10+19=29$

$\mathrm{w}[\mathrm{b}, \mathrm{v}, \mathrm{c}]=10+20=30$

$\mathrm{w}[\mathrm{b}, \mathrm{v}, \mathrm{r}]=10+20=30$

$\mathrm{w}[\mathrm{m}, \mathrm{v}, \mathrm{s}]=19+11=30$

$\mathrm{w}[\mathrm{c}, \mathrm{v}, \mathrm{s}]=20+11=31$

$\mathrm{w}[\mathrm{r}, \mathrm{v}, \mathrm{s}]=20+11=31$

$\mathrm{w}[\mathrm{c}, \mathrm{v}, \mathrm{m}]=20+19=39$

$\mathrm{w}[\mathrm{m}, \mathrm{v}, \mathrm{r}]=19+20=39$

$\mathrm{w}[\mathrm{c}, \mathrm{v}, \mathrm{r}]=20+20=40$

$\mathrm{w}[\mathrm{v}, \mathrm{s}, \mathrm{b}]=11+6=17$

$\mathrm{w}[\mathrm{b}, \mathrm{s}, \mathrm{c}]=6+15=21$

$\mathrm{w}[\mathrm{b}, \mathrm{s}, \mathrm{m}]=6+15=21$

$\mathrm{w}[\mathrm{b}, \mathrm{s}, \mathrm{r}]=6+15=21$

$\mathrm{w}[\mathrm{v}, \mathrm{s}, \mathrm{c}]=11+15=26$

$\mathrm{w}[\mathrm{v}, \mathrm{s}, \mathrm{m}]=11+15=26$

$\mathrm{w}[\mathrm{v}, \mathrm{s}, \mathrm{r}]=11+15=26$

$\mathrm{w}[\mathrm{c}, \mathrm{s}, \mathrm{m}]=15+15=30$

$\mathrm{w}[\mathrm{c}, \mathrm{s}, \mathrm{r}]=15+15=30$

$\mathrm{w}[\mathrm{m}, \mathrm{s}, \mathrm{r}]=15+15=30$

$\mathrm{w}[\mathrm{b}, \mathrm{r}, \mathrm{m}]=11+10=21$

$\mathrm{w}[\mathrm{s}, \mathrm{r}, \mathrm{m}]=15+10=25$

$\mathrm{w}[\mathrm{c}, \mathrm{r}, \mathrm{m}]=15+10=25$

$\mathrm{w}[\mathrm{s}, \mathrm{r}, \mathrm{b}]=15+11=26$

$\mathrm{w}[\mathrm{b}, \mathrm{r}, \mathrm{c}]=11+15=26$

$\mathrm{w}[\mathrm{s}, \mathrm{r}, \mathrm{c}]=15+15=30$

$\mathrm{w}[\mathrm{v}, \mathrm{r}, \mathrm{m}]=20+10=30$

$\mathrm{w}[\mathrm{v}, \mathrm{r}, \mathrm{b}]=20+11=31$

$\mathrm{w}[\mathrm{s}, \mathrm{r}, \mathrm{v}]=15+20=35$

$\mathrm{w}[\mathrm{v}, \mathrm{r}, \mathrm{c}]=20+15=35$

$\mathrm{w}[\mathrm{r}, \mathrm{m}, \mathrm{b}]=10+11=21$

$\mathrm{w}[\mathrm{r}, \mathrm{m}, \mathrm{s}]=10+15=25$

$\mathrm{w}[\mathrm{s}, \mathrm{m}, \mathrm{b}]=15+11=26$

$\mathrm{w}[\mathrm{r}, \mathrm{m}, \mathrm{c}]=10+17=27$

$\mathrm{w}[\mathrm{b}, \mathrm{m}, \mathrm{c}]=11+17=28$

$\mathrm{w}[\mathrm{r}, \mathrm{m}, \mathrm{v}]=10+19=29$

$\mathrm{w}[\mathrm{v}, \mathrm{m}, \mathrm{b}]=19+11=30$
$[\mathrm{m}, \mathrm{s}]+[\mathrm{m}, \mathrm{c}]=[\mathrm{s}, \mathrm{m}, \mathrm{c}]$
$[\mathrm{m}, \mathrm{s}]+[\mathrm{m}, \mathrm{v}]=[\mathrm{s}, \mathrm{m}, \mathrm{v}]$
$[\mathrm{m}, \mathrm{v}]+[\mathrm{m}, \mathrm{c}]=[\mathrm{v}, \mathrm{m}, \mathrm{c}]$
Vertex c (sorted)
$[\mathrm{c}, \mathrm{r}]+[\mathrm{c}, \mathrm{b}]=[\mathrm{r}, \mathrm{c}, \mathrm{b}]$
$[\mathrm{c}, \mathrm{s}]+[\mathrm{c}, \mathrm{b}]=[\mathrm{s}, \mathrm{c}, \mathrm{b}]$
$[\mathrm{c}, \mathrm{m}]+[\mathrm{c}, \mathrm{b}]=[\mathrm{m}, \mathrm{c}, \mathrm{b}]$
$[\mathrm{c}, \mathrm{r}]+[\mathrm{c}, \mathrm{s}]=[\mathrm{r}, \mathrm{c}, \mathrm{s}]$
$[\mathrm{c}, \mathrm{v}]+[\mathrm{c}, \mathrm{b}]=[\mathrm{v}, \mathrm{c}, \mathrm{b}]$
$[\mathrm{c}, \mathrm{m}]+[\mathrm{c}, \mathrm{r}]=[\mathrm{m}, \mathrm{c}, \mathrm{r}]$
$[\mathrm{c}, \mathrm{m}]+[\mathrm{c}, \mathrm{s}]=[\mathrm{m}, \mathrm{c}, \mathrm{s}]$
$[\mathrm{c}, \mathrm{r}]+[\mathrm{c}, \mathrm{v}]=[\mathrm{r}, \mathrm{c}, \mathrm{v}]$
$[\mathrm{c}, \mathrm{s}]+[\mathrm{c}, \mathrm{v}]=[\mathrm{s}, \mathrm{c}, \mathrm{v}]$
$[\mathrm{c}, \mathrm{m}]+[\mathrm{c}, \mathrm{v}]=[\mathrm{m}, \mathrm{c}, \mathrm{v}]$
$\mathrm{w}[\mathrm{s}, \mathrm{m}, \mathrm{c}]=15+17=32$
$\mathrm{w}[\mathrm{s}, \mathrm{m}, \mathrm{v}]=15+19=34$
$\mathrm{w}[\mathrm{v}, \mathrm{m}, \mathrm{c}]=19+17=36$
$\mathrm{w}[\mathrm{r}, \mathrm{c}, \mathrm{b}]=15+10=25$
$\mathrm{w}[\mathrm{s}, \mathrm{c}, \mathrm{b}]=15+10=25$
$\mathrm{w}[\mathrm{m}, \mathrm{c}, \mathrm{b}]=17+10=27$
$\mathrm{w}[\mathrm{r}, \mathrm{c}, \mathrm{s}]=15+15=30$
$\mathrm{w}[\mathrm{v}, \mathrm{c}, \mathrm{b}]=20+10=30$
$\mathrm{w}[\mathrm{m}, \mathrm{c}, \mathrm{r}]=17+15=32$
$\mathrm{w}[\mathrm{m}, \mathrm{c}, \mathrm{s}]=17+15=32$
$\mathrm{w}[\mathrm{r}, \mathrm{c}, \mathrm{v}]=15+20=35$
$\mathrm{w}[\mathrm{s}, \mathrm{c}, \mathrm{v}]=15+20=35$
$\mathrm{w}[\mathrm{m}, \mathrm{c}, \mathrm{v}]=17+20=37$

Section \#2.3 (END)

Section \#2.4 (BEGIN)

In this section, the partial circuit with the lowest weight under each vertex is taken individually and written below.
$[\mathrm{b}, \mathrm{c}]+[\mathrm{b}, \mathrm{s}]=[\mathrm{c}, \mathrm{b}, \mathrm{s}]$
$\mathrm{w}[\mathrm{c}, \mathrm{b}, \mathrm{s}]=10+6=16->\{\mathrm{D} 9\}$
$[\mathrm{v}, \mathrm{b}]+[\mathrm{v}, \mathrm{s}]=[\mathrm{b}, \mathrm{v}, \mathrm{s}]$
$\mathrm{w}[\mathrm{b}, \mathrm{v}, \mathrm{s}]=10+11=21->\{\mathrm{D} 10\}$
$[\mathrm{s}, \mathrm{v}]+[\mathrm{s}, \mathrm{b}]=[\mathrm{v}, \mathrm{s}, \mathrm{b}]$
$\mathrm{w}[\mathrm{v}, \mathrm{s}, \mathrm{b}]=11+6=17$
$[\mathrm{r}, \mathrm{b}]+[\mathrm{r}, \mathrm{m}]=[\mathrm{b}, \mathrm{r}, \mathrm{m}]$
$\mathrm{w}[\mathrm{b}, \mathrm{r}, \mathrm{m}]=11+10=21$
$[\mathrm{m}, \mathrm{r}]+[\mathrm{m}, \mathrm{b}]=[\mathrm{r}, \mathrm{m}, \mathrm{b}]$
$\mathrm{w}[\mathrm{r}, \mathrm{m}, \mathrm{b}]=10+11=21$
$[\mathrm{c}, \mathrm{r}]+[\mathrm{c}, \mathrm{b}]=[\mathrm{r}, \mathrm{c}, \mathrm{b}]$
$\mathrm{w}[\mathrm{r}, \mathrm{c}, \mathrm{b}]=15+10=25$

$->$ D9. Here $[c, b, s]$ is the lowest weight combination taken from Vertex b.

Vertex v.

$\rightarrow \mathrm{D} 10$. Here $[\mathrm{b}, \mathrm{v}, \mathrm{s}]$ is the lowest weight combination taken from

Section \#2.4 (END)

Section \#2.5 (BEGIN)

In this section, the partial circuits written in Section \#2.4 are sorted in 'Descending order' of their weights and written here.

$[\mathrm{c}, \mathrm{r}]+[\mathrm{c}, \mathrm{b}]=[\mathrm{r}, \mathrm{c}, \mathrm{b}] \quad \mathrm{w}[\mathrm{r}, \mathrm{c}, \mathrm{b}]=15+10=25($ Vertex $\mathrm{c})->\{\mathrm{D} 11\}$

$[\mathrm{v}, \mathrm{b}]+[\mathrm{v}, \mathrm{s}]=[\mathrm{b}, \mathrm{v}, \mathrm{s}] \quad \mathrm{w}[\mathrm{b}, \mathrm{v}, \mathrm{s}]=10+11=21$ (Vertex v)

$[\mathrm{r}, \mathrm{b}]+[\mathrm{r}, \mathrm{m}]=[\mathrm{b}, \mathrm{r}, \mathrm{m}] \quad \mathrm{w}[\mathrm{b}, \mathrm{r}, \mathrm{m}]=11+10=21$ (Vertex $\mathrm{r})$

$[\mathrm{m}, \mathrm{r}]+[\mathrm{m}, \mathrm{b}]=[\mathrm{r}, \mathrm{m}, \mathrm{b}] \quad \mathrm{w}[\mathrm{r}, \mathrm{m}, \mathrm{b}]=10+11=21($ Vertex $\mathrm{m})$

$[\mathrm{s}, \mathrm{v}]+[\mathrm{s}, \mathrm{b}]=[\mathrm{v}, \mathrm{s}, \mathrm{b}] \quad \mathrm{w}[\mathrm{v}, \mathrm{s}, \mathrm{b}]=11+6=17 \quad($ Vertex s)

$[\mathrm{b}, \mathrm{c}]+[\mathrm{b}, \mathrm{s}]=[\mathrm{c}, \mathrm{b}, \mathrm{s}] \quad \mathrm{w}[\mathrm{c}, \mathrm{b}, \mathrm{s}]=10+6=16 \quad($ Vertex $\mathrm{b})$

$->\mathrm{D} 11$. $[\mathrm{r}, \mathrm{c}, \mathrm{b}]$ is a partial circuit having the highest weight in the group in this section.

Section \#2.5 (END)

Section \#2.6 (BEGIN) 
In this section, in order to choose a partial circuit ( or the order of which vertex to choose from ) is given by the order in Section \#2.5. So the order is as follows ie:- vertex $\mathrm{c}$, vertex $\mathrm{v}$, vertex $\mathrm{r}$, vertex $\mathrm{m}$, vertex $s$, vertex b.

$$
\begin{aligned}
& \text { Vertex c (sorted) } \\
& {[\mathrm{c}, \mathrm{r}]+[\mathrm{c}, \mathrm{b}]=[\mathrm{r}, \mathrm{c}, \mathrm{b}]} \\
& {[\mathrm{c}, \mathrm{s}]+[\mathrm{c}, \mathrm{b}]=[\mathrm{s}, \mathrm{c}, \mathrm{b}]} \\
& {[\mathrm{c}, \mathrm{m}]+[\mathrm{c}, \mathrm{b}]=[\mathrm{m}, \mathrm{c}, \mathrm{b}]} \\
& {[\mathrm{c}, \mathrm{r}]+[\mathrm{c}, \mathrm{s}]=[\mathrm{r}, \mathrm{c}, \mathrm{s}]} \\
& {[\mathrm{c}, \mathrm{v}]+[\mathrm{c}, \mathrm{b}]=[\mathrm{v}, \mathrm{c}, \mathrm{b}]} \\
& {[\mathrm{c}, \mathrm{m}]+[\mathrm{c}, \mathrm{r}]=[\mathrm{m}, \mathrm{c}, \mathrm{r}]} \\
& {[\mathrm{c}, \mathrm{m}]+[\mathrm{c}, \mathrm{s}]=[\mathrm{m}, \mathrm{c}, \mathrm{s}]} \\
& {[\mathrm{c}, \mathrm{r}]+[\mathrm{c}, \mathrm{v}]=[\mathrm{r}, \mathrm{c}, \mathrm{v}]} \\
& {[\mathrm{c}, \mathrm{s}]+[\mathrm{c}, \mathrm{v}]=[\mathrm{s}, \mathrm{c}, \mathrm{v}]} \\
& {[\mathrm{c}, \mathrm{m}]+[\mathrm{c}, \mathrm{v}]=[\mathrm{m}, \mathrm{c}, \mathrm{v}]}
\end{aligned}
$$

Vertex v (sorted)

$[\mathrm{v}, \mathrm{b}]+[\mathrm{v}, \mathrm{s}]=[\mathrm{b}, \mathrm{v}, \mathrm{s}]$

$[\mathrm{v}, \mathrm{b}]+[\mathrm{v}, \mathrm{m}]=[\mathrm{b}, \mathrm{v}, \mathrm{m}]$

$[\mathrm{v}, \mathrm{b}]+[\mathrm{v}, \mathrm{c}]=[\mathrm{b}, \mathrm{v}, \mathrm{c}]$

$[\mathrm{v}, \mathrm{b}]+[\mathrm{v}, \mathrm{r}]=[\mathrm{b}, \mathrm{v}, \mathrm{r}]$

$[\mathrm{v}, \mathrm{m}]+[\mathrm{v}, \mathrm{s}]=[\mathrm{m}, \mathrm{v}, \mathrm{s}]$

$[\mathrm{v}, \mathrm{c}]+[\mathrm{v}, \mathrm{s}]=[\mathrm{c}, \mathrm{v}, \mathrm{s}]$

$[\mathrm{v}, \mathrm{r}]+[\mathrm{v}, \mathrm{s}]=[\mathrm{r}, \mathrm{v}, \mathrm{s}]$

$[\mathrm{v}, \mathrm{c}]+[\mathrm{v}, \mathrm{m}]=[\mathrm{c}, \mathrm{v}, \mathrm{m}]$

$[\mathrm{v}, \mathrm{m}]+[\mathrm{v}, \mathrm{r}]=[\mathrm{m}, \mathrm{v}, \mathrm{r}]$

$[\mathrm{v}, \mathrm{c}]+[\mathrm{v}, \mathrm{r}]=[\mathrm{c}, \mathrm{v}, \mathrm{r}]$

Vertex r (sorted)

$[\mathrm{r}, \mathrm{b}]+[\mathrm{r}, \mathrm{m}]=[\mathrm{b}, \mathrm{r}, \mathrm{m}]$

$[\mathrm{r}, \mathrm{s}]+[\mathrm{r}, \mathrm{m}]=[\mathrm{s}, \mathrm{r}, \mathrm{m}]$

$[\mathrm{r}, \mathrm{c}]+[\mathrm{r}, \mathrm{m}]=[\mathrm{c}, \mathrm{r}, \mathrm{m}]$

$[\mathrm{r}, \mathrm{s}]+[\mathrm{r}, \mathrm{b}]=[\mathrm{s}, \mathrm{r}, \mathrm{b}]$

$[\mathrm{r}, \mathrm{b}]+[\mathrm{r}, \mathrm{c}]=[\mathrm{b}, \mathrm{r}, \mathrm{c}]$

$[\mathrm{r}, \mathrm{s}]+[\mathrm{r}, \mathrm{c}]=[\mathrm{s}, \mathrm{r}, \mathrm{c}]$

$[\mathrm{r}, \mathrm{v}]+[\mathrm{r}, \mathrm{m}]=[\mathrm{v}, \mathrm{r}, \mathrm{m}]$

$[\mathrm{r}, \mathrm{v}]+[\mathrm{r}, \mathrm{b}]=[\mathrm{v}, \mathrm{r}, \mathrm{b}]$

$[\mathrm{r}, \mathrm{s}]+[\mathrm{r}, \mathrm{v}]=[\mathrm{s}, \mathrm{r}, \mathrm{v}]$

$[\mathrm{r}, \mathrm{v}]+[\mathrm{r}, \mathrm{c}]=[\mathrm{v}, \mathrm{r}, \mathrm{c}]$

Vertex m (sorted)

$[\mathrm{m}, \mathrm{r}]+[\mathrm{m}, \mathrm{b}]=[\mathrm{r}, \mathrm{m}, \mathrm{b}]$

$[\mathrm{m}, \mathrm{r}]+[\mathrm{m}, \mathrm{s}]=[\mathrm{r}, \mathrm{m}, \mathrm{s}]$

$[\mathrm{m}, \mathrm{s}]+[\mathrm{m}, \mathrm{b}]=[\mathrm{s}, \mathrm{m}, \mathrm{b}]$

$$
\begin{aligned}
& \mathrm{w}[\mathrm{r}, \mathrm{c}, \mathrm{b}]=15+10=25 \\
& \mathrm{w}[\mathrm{s}, \mathrm{c}, \mathrm{b}]=15+10=25 \\
& \mathrm{w}[\mathrm{m}, \mathrm{c}, \mathrm{b}]=17+10=27 \\
& \mathrm{w}[\mathrm{r}, \mathrm{c}, \mathrm{s}]=15+15=30 \\
& \mathrm{w}[\mathrm{v}, \mathrm{c}, \mathrm{b}]=20+10=30 \\
& \mathrm{w}[\mathrm{m}, \mathrm{c}, \mathrm{r}]=17+15=32 \\
& \mathrm{w}[\mathrm{m}, \mathrm{c}, \mathrm{s}]=17+15=32 \\
& \mathrm{w}[\mathrm{r}, \mathrm{c}, \mathrm{v}]=15+20=35 \\
& \mathrm{w}[\mathrm{s}, \mathrm{c}, \mathrm{v}]=15+20=35 \\
& \mathrm{w}[\mathrm{m}, \mathrm{c}, \mathrm{v}]=17+20=37
\end{aligned}
$$

$\mathrm{w}[\mathrm{b}, \mathrm{v}, \mathrm{s}]=10+11=21$

$\mathrm{w}[\mathrm{b}, \mathrm{v}, \mathrm{m}]=10+19=29$

$\mathrm{w}[\mathrm{b}, \mathrm{v}, \mathrm{c}]=10+20=30$

$\mathrm{w}[\mathrm{b}, \mathrm{v}, \mathrm{r}]=10+20=30$

$\mathrm{w}[\mathrm{m}, \mathrm{v}, \mathrm{s}]=19+11=30$

$\mathrm{w}[\mathrm{c}, \mathrm{v}, \mathrm{s}]=20+11=31$

$\mathrm{w}[\mathrm{r}, \mathrm{v}, \mathrm{s}]=20+11=31$

$\mathrm{w}[\mathrm{c}, \mathrm{v}, \mathrm{m}]=20+19=39$

$\mathrm{w}[\mathrm{m}, \mathrm{v}, \mathrm{r}]=19+20=39$

$\mathrm{w}[\mathrm{c}, \mathrm{v}, \mathrm{r}]=20+20=40$

$\mathrm{w}[\mathrm{b}, \mathrm{r}, \mathrm{m}]=11+10=21$

$\mathrm{w}[\mathrm{s}, \mathrm{r}, \mathrm{m}]=15+10=25$

$\mathrm{w}[\mathrm{c}, \mathrm{r}, \mathrm{m}]=15+10=25$

$\mathrm{w}[\mathrm{s}, \mathrm{r}, \mathrm{b}]=15+11=26$

$\mathrm{w}[\mathrm{b}, \mathrm{r}, \mathrm{c}]=11+15=26$

$\mathrm{w}[\mathrm{s}, \mathrm{r}, \mathrm{c}]=15+15=30$

$\mathrm{w}[\mathrm{v}, \mathrm{r}, \mathrm{m}]=20+10=30$

$\mathrm{w}[\mathrm{v}, \mathrm{r}, \mathrm{b}]=20+11=31$

$\mathrm{w}[\mathrm{s}, \mathrm{r}, \mathrm{v}]=15+20=35$

$\mathrm{w}[\mathrm{v}, \mathrm{r}, \mathrm{c}]=20+15=35$

$\mathrm{w}[\mathrm{r}, \mathrm{m}, \mathrm{b}]=10+11=21$

$\mathrm{w}[\mathrm{r}, \mathrm{m}, \mathrm{s}]=10+15=25$

$\mathrm{w}[\mathrm{s}, \mathrm{m}, \mathrm{b}]=15+11=26$

$$
\begin{aligned}
& {[\mathrm{m}, \mathrm{r}]+[\mathrm{m}, \mathrm{c}]=[\mathrm{r}, \mathrm{m}, \mathrm{c}]} \\
& {[\mathrm{m}, \mathrm{b}]+[\mathrm{m}, \mathrm{c}]=[\mathrm{b}, \mathrm{m}, \mathrm{c}]} \\
& {[\mathrm{m}, \mathrm{r}]+[\mathrm{m}, \mathrm{v}]=[\mathrm{r}, \mathrm{m}, \mathrm{v}]} \\
& {[\mathrm{m}, \mathrm{v}]+[\mathrm{m}, \mathrm{b}]=[\mathrm{v}, \mathrm{m}, \mathrm{b}]} \\
& {[\mathrm{m}, \mathrm{s}]+[\mathrm{m}, \mathrm{c}]=[\mathrm{s}, \mathrm{m}, \mathrm{c}]} \\
& {[\mathrm{m}, \mathrm{s}]+[\mathrm{m}, \mathrm{v}]=[\mathrm{s}, \mathrm{m}, \mathrm{v}]} \\
& {[\mathrm{m}, \mathrm{v}]+[\mathrm{m}, \mathrm{c}]=[\mathrm{v}, \mathrm{m}, \mathrm{c}]}
\end{aligned}
$$$$
\text { Vertex s (sorted) }
$$$$
[\mathrm{s}, \mathrm{v}]+[\mathrm{s}, \mathrm{b}]=[\mathrm{v}, \mathrm{s}, \mathrm{b}]
$$$$
[\mathrm{s}, \mathrm{b}]+[\mathrm{s}, \mathrm{c}]=[\mathrm{b}, \mathrm{s}, \mathrm{c}]
$$$$
[\mathrm{s}, \mathrm{b}]+[\mathrm{s}, \mathrm{m}]=[\mathrm{b}, \mathrm{s}, \mathrm{m}]
$$$$
[\mathrm{s}, \mathrm{b}]+[\mathrm{s}, \mathrm{r}]=[\mathrm{b}, \mathrm{s}, \mathrm{r}]
$$$$
[\mathrm{s}, \mathrm{v}]+[\mathrm{s}, \mathrm{c}]=[\mathrm{v}, \mathrm{s}, \mathrm{c}]
$$$$
[\mathrm{s}, \mathrm{v}]+[\mathrm{s}, \mathrm{m}]=[\mathrm{v}, \mathrm{s}, \mathrm{m}]
$$$$
[\mathrm{s}, \mathrm{v}]+[\mathrm{s}, \mathrm{r}]=[\mathrm{v}, \mathrm{s}, \mathrm{r}]
$$$$
[\mathrm{s}, \mathrm{c}]+[\mathrm{s}, \mathrm{m}]=[\mathrm{c}, \mathrm{s}, \mathrm{m}]
$$$$
[\mathrm{s}, \mathrm{c}]+[\mathrm{s}, \mathrm{r}]=[\mathrm{c}, \mathrm{s}, \mathrm{r}]
$$$$
[\mathrm{s}, \mathrm{m}]+[\mathrm{s}, \mathrm{r}]=[\mathrm{m}, \mathrm{s}, \mathrm{r}]
$$$$
\text { Vertex b (sorted) }
$$$$
[b, c]+[b, s]=[c, b, s]
$$$$
[\mathrm{b}, \mathrm{s}]+[\mathrm{b}, \mathrm{v}]=[\mathrm{s}, \mathrm{b}, \mathrm{v}]
$$$$
[\mathrm{b}, \mathrm{m}]+[\mathrm{b}, \mathrm{s}]=[\mathrm{m}, \mathrm{b}, \mathrm{s}]
$$$$
[\mathrm{b}, \mathrm{r}]+[\mathrm{b}, \mathrm{s}]=[\mathrm{r}, \mathrm{b}, \mathrm{s}]
$$$$
[\mathrm{b}, \mathrm{c}]+[\mathrm{b}, \mathrm{v}]=[\mathrm{c}, \mathrm{b}, \mathrm{v}]
$$$$
[\mathrm{b}, \mathrm{c}]+[\mathrm{b}, \mathrm{m}]=[\mathrm{c}, \mathrm{b}, \mathrm{m}]
$$$$
[\mathrm{b}, \mathrm{c}]+[\mathrm{b}, \mathrm{r}]=[\mathrm{c}, \mathrm{b}, \mathrm{r}]
$$$$
[\mathrm{b}, \mathrm{m}]+[\mathrm{b}, \mathrm{v}]=[\mathrm{m}, \mathrm{b}, \mathrm{v}]
$$$$
[\mathrm{b}, \mathrm{r}]+[\mathrm{b}, \mathrm{v}]=[\mathrm{r}, \mathrm{b}, \mathrm{v}]
$$$$
[\mathrm{b}, \mathrm{m}]+[\mathrm{b}, \mathrm{r}]=[\mathrm{m}, \mathrm{b}, \mathrm{r}]
$$$$
\mathrm{w}[\mathrm{v}, \mathrm{s}, \mathrm{b}]=11+6=17
$$$$
\mathrm{w}[\mathrm{b}, \mathrm{s}, \mathrm{c}]=6+15=21
$$$$
\mathrm{w}[\mathrm{b}, \mathrm{s}, \mathrm{m}]=6+15=21
$$$$
\mathrm{w}[\mathrm{b}, \mathrm{s}, \mathrm{r}]=6+15=21
$$$$
\mathrm{w}[\mathrm{v}, \mathrm{s}, \mathrm{c}]=11+15=26
$$$$
\mathrm{w}[\mathrm{v}, \mathrm{s}, \mathrm{m}]=11+15=26
$$$$
\mathrm{w}[\mathrm{v}, \mathrm{s}, \mathrm{r}]=11+15=26
$$$$
\mathrm{w}[\mathrm{c}, \mathrm{s}, \mathrm{m}]=15+15=30
$$$$
\mathrm{w}[\mathrm{c}, \mathrm{s}, \mathrm{r}]=15+15=30
$$$$
\mathrm{w}[\mathrm{m}, \mathrm{s}, \mathrm{r}]=15+15=30
$$

$\mathrm{w}[\mathrm{c}, \mathrm{b}, \mathrm{s}]=10+6=16$ $\mathrm{w}[\mathrm{s}, \mathrm{b}, \mathrm{v}]=6+10=16$ $\mathrm{w}[\mathrm{m}, \mathrm{b}, \mathrm{s}]=11+6=17$ $\mathrm{w}[\mathrm{r}, \mathrm{b}, \mathrm{s}]=11+6=17$ $\mathrm{w}[\mathrm{c}, \mathrm{b}, \mathrm{v}]=10+10=20$ $\mathrm{w}[\mathrm{c}, \mathrm{b}, \mathrm{m}]=10+11=21$ $\mathrm{w}[\mathrm{c}, \mathrm{b}, \mathrm{r}]=10+11=21$ $\mathrm{w}[\mathrm{m}, \mathrm{b}, \mathrm{v}]=11+10=21$ $\mathrm{w}[\mathrm{r}, \mathrm{b}, \mathrm{v}]=11+10=21$

$\mathrm{w}[\mathrm{m}, \mathrm{b}, \mathrm{r}]=11+11=22$

Section \#2.6 (END)

Section \#2.7 (BEGIN)

Now from Section \#2.6, take a partial circuit from each vertex (the order of the vertex to be taken, should be the same order in which the vertices are written in Section \#2.6) and join them together in order to form a hamilton circuit. The process is as follows:-

1) Take $[r, c, b]$ from Vertex c. Since hamilton circuit rule is not violated, $[r, c, b]$ is taken.

2) Take $[b, v, s]$ from Vertex v. $[b, v, s]$ fits with $[r, c, b]$. So $[b, v, s]$ is also taken. The new partial circuit is $[\mathrm{r}, \mathrm{c}, \mathrm{b}, \mathrm{v}, \mathrm{s}]$.

3) Take $[b, r, m]$ from Vertex r. [b,r,m] does not fit with $[r, c, b, v, s]$. So the next step is not to go to the next vertex, but to take another partial circuit from the same vertex to see if it fits with $[\mathrm{r}, \mathrm{c}, \mathrm{b}, \mathrm{v}, \mathrm{s}]$. So take $[\mathrm{s}, \mathrm{r}, \mathrm{m}]$ from Vertex $\mathrm{r}$. [s,r,m] does not fit with [r,c,b,v,s]. So take 
the next partial circuit from the same vertex to see if fits with [r,c,b,v,s]. So take $[c, r, m]$ from Vertex r. [c,r,m] fits with [r,c,b,v,s]. Fitting [c,r,m] with $[\mathrm{r}, \mathrm{c}, \mathrm{b}, \mathrm{v}, \mathrm{s}]$ gives the partial circuit $[\mathrm{m}, \mathrm{r}, \mathrm{c}, \mathrm{b}, \mathrm{v}, \mathrm{s}]$.

4) Take $[r, m, b]$ from Vertex $m$. [r,m,b] does not fit with $[\mathrm{m}, \mathrm{r}, \mathrm{c}, \mathrm{b}, \mathrm{v}, \mathrm{s}]$. Take $[\mathrm{r}, \mathrm{m}, \mathrm{s}]$ from vertex $\mathrm{m}$. [r,m,s] fits with $[\mathrm{m}, \mathrm{r}, \mathrm{c}, \mathrm{b}, \mathrm{v}, \mathrm{s}]$ to create the hamilton circuit $[\mathrm{r}, \mathrm{c}, \mathrm{b}, \mathrm{v}, \mathrm{s}, \mathrm{m}, \mathrm{r}]$. The weight of the hamilton circuit $\mathrm{w}[\mathrm{r}, \mathrm{c}, \mathrm{b}, \mathrm{v}, \mathrm{s}, \mathrm{m}, \mathrm{r}]=\mathrm{w}[\mathrm{r}, \mathrm{c}]+\mathrm{w}[\mathrm{c}, \mathrm{b}]+\mathrm{w}[\mathrm{b}, \mathrm{v}]+\mathrm{w}[\mathrm{v}, \mathrm{s}]+\mathrm{w}[\mathrm{s}, \mathrm{m}]+$ $\mathrm{w}[\mathrm{m}, \mathrm{r}]=15+10+10+11+15+10=71$.

So $[\mathrm{r}, \mathrm{c}, \mathrm{b}, \mathrm{v}, \mathrm{s}, \mathrm{m}, \mathrm{r}]=[\mathrm{r}, \mathrm{c}, \mathrm{b}]+[\mathrm{b}, \mathrm{v}, \mathrm{s}]+[\mathrm{c}, \mathrm{r}, \mathrm{m}]+[\mathrm{r}, \mathrm{m}, \mathrm{s}]$.

$\mathrm{w}[\mathrm{r}, \mathrm{c}, \mathrm{b}, \mathrm{v}, \mathrm{s}, \mathrm{m}, \mathrm{r}]=71$

The hamilton circuit was obtained, and to know if it is the minimum weight hamilton circuit, checking is needed to see if any lower partial circuits which have been bypassed will form lesser weight hamilton circuits. To know which all are the lesser weight partial circuits, the next section ( ie:- Section \#2.8 ) onwards shows the lesser weight partial circuits.

Section \#2.7 (END)

Section \#2.8 (BEGIN)

Group the partial circuits of the different vertices present in Section \#2.6 into one group and then sort it. So first grouping the partial circuits, we obtain as below:-

$$
\begin{aligned}
& {[\mathrm{c}, \mathrm{r}]+[\mathrm{c}, \mathrm{b}]=[\mathrm{r}, \mathrm{c}, \mathrm{b}]} \\
& {[\mathrm{c}, \mathrm{s}]+[\mathrm{c}, \mathrm{b}]=[\mathrm{s}, \mathrm{c}, \mathrm{b}]} \\
& {[\mathrm{c}, \mathrm{m}]+[\mathrm{c}, \mathrm{b}]=[\mathrm{m}, \mathrm{c}, \mathrm{b}]} \\
& {[\mathrm{c}, \mathrm{r}]+[\mathrm{c}, \mathrm{s}]=[\mathrm{r}, \mathrm{c}, \mathrm{s}]} \\
& {[\mathrm{c}, \mathrm{v}]+[\mathrm{c}, \mathrm{b}]=[\mathrm{v}, \mathrm{c}, \mathrm{b}]} \\
& {[\mathrm{c}, \mathrm{m}]+[\mathrm{c}, \mathrm{r}]=[\mathrm{m}, \mathrm{c}, \mathrm{r}]} \\
& {[\mathrm{c}, \mathrm{m}]+[\mathrm{c}, \mathrm{s}]=[\mathrm{m}, \mathrm{c}, \mathrm{s}]} \\
& {[\mathrm{c}, \mathrm{r}]+[\mathrm{c}, \mathrm{v}]=[\mathrm{r}, \mathrm{c}, \mathrm{v}]} \\
& {[\mathrm{c}, \mathrm{s}]+[\mathrm{c}, \mathrm{v}]=[\mathrm{s}, \mathrm{c}, \mathrm{v}]} \\
& {[\mathrm{c}, \mathrm{m}]+[\mathrm{c}, \mathrm{v}]=[\mathrm{m}, \mathrm{c}, \mathrm{v}]} \\
& {[\mathrm{v}, \mathrm{b}]+[\mathrm{v}, \mathrm{s}]=[\mathrm{b}, \mathrm{v}, \mathrm{s}]} \\
& {[\mathrm{v}, \mathrm{b}]+[\mathrm{v}, \mathrm{m}]=[\mathrm{b}, \mathrm{v}, \mathrm{m}]} \\
& {[\mathrm{v}, \mathrm{b}]+[\mathrm{v}, \mathrm{c}]=[\mathrm{b}, \mathrm{v}, \mathrm{c}]} \\
& {[\mathrm{v}, \mathrm{b}]+[\mathrm{v}, \mathrm{r}]=[\mathrm{b}, \mathrm{v}, \mathrm{r}]} \\
& {[\mathrm{v}, \mathrm{m}]+[\mathrm{v}, \mathrm{s}]=[\mathrm{m}, \mathrm{v}, \mathrm{s}]} \\
& {[\mathrm{v}, \mathrm{c}]+[\mathrm{v}, \mathrm{s}]=[\mathrm{c}, \mathrm{v}, \mathrm{s}]} \\
& {[\mathrm{v}, \mathrm{r}]+[\mathrm{v}, \mathrm{s}]=[\mathrm{r}, \mathrm{v}, \mathrm{s}]} \\
& {[\mathrm{r}, \mathrm{s}]+[\mathrm{r}, \mathrm{b}]=[\mathrm{s}, \mathrm{r}, \mathrm{b}]} \\
& {[\mathrm{v}, \mathrm{c}]+[\mathrm{v}, \mathrm{m}]=[\mathrm{c}, \mathrm{v}, \mathrm{m}]} \\
& {[\mathrm{v}, \mathrm{m}]+[\mathrm{v}, \mathrm{r}]=[\mathrm{m}, \mathrm{v}, \mathrm{r}]} \\
& {[\mathrm{v}, \mathrm{c}]+[\mathrm{v}, \mathrm{r}]=[\mathrm{c}, \mathrm{v}, \mathrm{r}]} \\
&
\end{aligned}
$$

$$
\begin{aligned}
& {[\mathrm{r}, \mathrm{b}]+[\mathrm{r}, \mathrm{c}]=[\mathrm{b}, \mathrm{r}, \mathrm{c}]} \\
& {[\mathrm{r}, \mathrm{s}]+[\mathrm{r}, \mathrm{c}]=[\mathrm{s}, \mathrm{r}, \mathrm{c}]} \\
& {[\mathrm{r}, \mathrm{v}]+[\mathrm{r}, \mathrm{m}]=[\mathrm{v}, \mathrm{r}, \mathrm{m}]} \\
& {[\mathrm{r}, \mathrm{v}]+[\mathrm{r}, \mathrm{b}]=[\mathrm{v}, \mathrm{r}, \mathrm{b}]} \\
& {[\mathrm{r}, \mathrm{s}]+[\mathrm{r}, \mathrm{v}]=[\mathrm{s}, \mathrm{r}, \mathrm{v}]} \\
& {[\mathrm{r}, \mathrm{v}]+[\mathrm{r}, \mathrm{c}]=[\mathrm{v}, \mathrm{r}, \mathrm{c}]} \\
& {[\mathrm{m}, \mathrm{r}]+[\mathrm{m}, \mathrm{b}]=[\mathrm{r}, \mathrm{m}, \mathrm{b}]} \\
& {[\mathrm{m}, \mathrm{r}]+[\mathrm{m}, \mathrm{s}]=[\mathrm{r}, \mathrm{m}, \mathrm{s}]} \\
& {[\mathrm{m}, \mathrm{s}]+[\mathrm{m}, \mathrm{b}]=[\mathrm{s}, \mathrm{m}, \mathrm{b}]} \\
& {[\mathrm{m}, \mathrm{r}]+[\mathrm{m}, \mathrm{c}]=[\mathrm{r}, \mathrm{m}, \mathrm{c}]} \\
& {[\mathrm{m}, \mathrm{b}]+[\mathrm{m}, \mathrm{c}]=[\mathrm{b}, \mathrm{m}, \mathrm{c}]} \\
& {[\mathrm{m}, \mathrm{r}]+[\mathrm{m}, \mathrm{v}]=[\mathrm{r}, \mathrm{m}, \mathrm{v}]} \\
& {[\mathrm{m}, \mathrm{v}]+[\mathrm{m}, \mathrm{b}]=[\mathrm{v}, \mathrm{m}, \mathrm{b}]} \\
& {[\mathrm{m}, \mathrm{s}]+[\mathrm{m}, \mathrm{c}]=[\mathrm{s}, \mathrm{m}, \mathrm{c}]} \\
& {[\mathrm{m}, \mathrm{s}]+[\mathrm{m}, \mathrm{v}]=[\mathrm{s}, \mathrm{m}, \mathrm{v}]} \\
& {[\mathrm{m}, \mathrm{v}]+[\mathrm{m}, \mathrm{c}]=[\mathrm{v}, \mathrm{m}, \mathrm{c}]} \\
& {[\mathrm{s}, \mathrm{v}]+[\mathrm{s}, \mathrm{b}]=[\mathrm{v}, \mathrm{s}, \mathrm{b}]} \\
& {[\mathrm{s}, \mathrm{b}]+[\mathrm{s}, \mathrm{c}]=[\mathrm{b}, \mathrm{s}, \mathrm{c}]} \\
& {[\mathrm{s}, \mathrm{b}]+[\mathrm{s}, \mathrm{m}]=[\mathrm{b}, \mathrm{s}, \mathrm{m}]} \\
& {[\mathrm{s}, \mathrm{b}]+[\mathrm{s}, \mathrm{r}]=[\mathrm{b}, \mathrm{s}, \mathrm{r}]} \\
& {[\mathrm{s}, \mathrm{v}]+[\mathrm{s}, \mathrm{c}]=[\mathrm{v}, \mathrm{s}, \mathrm{c}]} \\
& {[\mathrm{s}, \mathrm{v}]+[\mathrm{s}, \mathrm{m}]=[\mathrm{v}, \mathrm{s}, \mathrm{m}]} \\
& {[\mathrm{s}, \mathrm{v}]+[\mathrm{s}, \mathrm{r}]=[\mathrm{v}, \mathrm{s}, \mathrm{r}]} \\
& {[\mathrm{s}, \mathrm{c}]+[\mathrm{s}, \mathrm{m}]=[\mathrm{c}, \mathrm{s}, \mathrm{m}]} \\
& {[\mathrm{s}, \mathrm{c}]+[\mathrm{s}, \mathrm{r}]=[\mathrm{c}, \mathrm{s}, \mathrm{r}]} \\
& {[\mathrm{s}, \mathrm{m}]+[\mathrm{s}, \mathrm{r}]=[\mathrm{m}, \mathrm{s}, \mathrm{r}]} \\
& {[b, c]+[b, s]=[c, b, s]} \\
& {[\mathrm{b}, \mathrm{s}]+[\mathrm{b}, \mathrm{v}]=[\mathrm{s}, \mathrm{b}, \mathrm{v}]} \\
& {[\mathrm{b}, \mathrm{m}]+[\mathrm{b}, \mathrm{s}]=[\mathrm{m}, \mathrm{b}, \mathrm{s}]} \\
& {[\mathrm{b}, \mathrm{r}]+[\mathrm{b}, \mathrm{s}]=[\mathrm{r}, \mathrm{b}, \mathrm{s}]} \\
& {[\mathrm{b}, \mathrm{c}]+[\mathrm{b}, \mathrm{v}]=[\mathrm{c}, \mathrm{b}, \mathrm{v}]} \\
& {[\mathrm{b}, \mathrm{c}]+[\mathrm{b}, \mathrm{m}]=[\mathrm{c}, \mathrm{b}, \mathrm{m}]} \\
& {[\mathrm{b}, \mathrm{c}]+[\mathrm{b}, \mathrm{r}]=[\mathrm{c}, \mathrm{b}, \mathrm{r}]} \\
& {[\mathrm{b}, \mathrm{m}]+[\mathrm{b}, \mathrm{v}]=[\mathrm{m}, \mathrm{b}, \mathrm{v}]} \\
& {[\mathrm{b}, \mathrm{r}]+[\mathrm{b}, \mathrm{v}]=[\mathrm{r}, \mathrm{b}, \mathrm{v}]} \\
& {[\mathrm{b}, \mathrm{m}]+[\mathrm{b}, \mathrm{r}]=[\mathrm{m}, \mathrm{b}, \mathrm{r}]}
\end{aligned}
$$$$
\text { Section \#2.8 (END) }
$$$$
\text { Section \#2.9 (BEGIN) }
$$

Now sort the group obtained in Section \#2.8 according to their weights. The sorted list of the different partial circuits is as follows. 


$$
\begin{aligned}
& {[\mathrm{b}, \mathrm{c}]+[\mathrm{b}, \mathrm{s}]=[\mathrm{c}, \mathrm{b}, \mathrm{s}]} \\
& {[\mathrm{b}, \mathrm{s}]+[\mathrm{b}, \mathrm{v}]=[\mathrm{s}, \mathrm{b}, \mathrm{v}]} \\
& {[\mathrm{s}, \mathrm{v}]+[\mathrm{s}, \mathrm{b}]=[\mathrm{v}, \mathrm{s}, \mathrm{b}]} \\
& {[\mathrm{b}, \mathrm{m}]+[\mathrm{b}, \mathrm{s}]=[\mathrm{m}, \mathrm{b}, \mathrm{s}]} \\
& {[\mathrm{b}, \mathrm{r}]+[\mathrm{b}, \mathrm{s}]=[\mathrm{r}, \mathrm{b}, \mathrm{s}]} \\
& {[b, c]+[b, v]=[c, b, v]} \\
& {[\mathrm{v}, \mathrm{b}]+[\mathrm{v}, \mathrm{s}]=[\mathrm{b}, \mathrm{v}, \mathrm{s}]} \\
& {[\mathrm{r}, \mathrm{b}]+[\mathrm{r}, \mathrm{m}]=[\mathrm{b}, \mathrm{r}, \mathrm{m}]} \\
& {[\mathrm{m}, \mathrm{r}]+[\mathrm{m}, \mathrm{b}]=[\mathrm{r}, \mathrm{m}, \mathrm{b}]} \\
& {[\mathrm{s}, \mathrm{b}]+[\mathrm{s}, \mathrm{c}]=[\mathrm{b}, \mathrm{s}, \mathrm{c}]} \\
& {[\mathrm{s}, \mathrm{b}]+[\mathrm{s}, \mathrm{m}]=[\mathrm{b}, \mathrm{s}, \mathrm{m}]} \\
& {[\mathrm{s}, \mathrm{b}]+[\mathrm{s}, \mathrm{r}]=[\mathrm{b}, \mathrm{s}, \mathrm{r}]} \\
& {[\mathrm{b}, \mathrm{c}]+[\mathrm{b}, \mathrm{m}]=[\mathrm{c}, \mathrm{b}, \mathrm{m}]} \\
& {[\mathrm{b}, \mathrm{c}]+[\mathrm{b}, \mathrm{r}]=[\mathrm{c}, \mathrm{b}, \mathrm{r}]} \\
& {[\mathrm{b}, \mathrm{m}]+[\mathrm{b}, \mathrm{v}]=[\mathrm{m}, \mathrm{b}, \mathrm{v}]} \\
& {[\mathrm{b}, \mathrm{r}]+[\mathrm{b}, \mathrm{v}]=[\mathrm{r}, \mathrm{b}, \mathrm{v}]} \\
& {[\mathrm{b}, \mathrm{m}]+[\mathrm{b}, \mathrm{r}]=[\mathrm{m}, \mathrm{b}, \mathrm{r}]} \\
& {[\mathrm{c}, \mathrm{r}]+[\mathrm{c}, \mathrm{b}]=[\mathrm{r}, \mathrm{c}, \mathrm{b}]} \\
& {[\mathrm{c}, \mathrm{s}]+[\mathrm{c}, \mathrm{b}]=[\mathrm{s}, \mathrm{c}, \mathrm{b}]} \\
& {[\mathrm{r}, \mathrm{s}]+[\mathrm{r}, \mathrm{m}]=[\mathrm{s}, \mathrm{r}, \mathrm{m}]} \\
& {[\mathrm{r}, \mathrm{c}]+[\mathrm{r}, \mathrm{m}]=[\mathrm{c}, \mathrm{r}, \mathrm{m}]} \\
& {[\mathrm{m}, \mathrm{r}]+[\mathrm{m}, \mathrm{s}]=[\mathrm{r}, \mathrm{m}, \mathrm{s}]} \\
& {[\mathrm{r}, \mathrm{s}]+[\mathrm{r}, \mathrm{b}]=[\mathrm{s}, \mathrm{r}, \mathrm{b}]} \\
& {[\mathrm{r}, \mathrm{b}]+[\mathrm{r}, \mathrm{c}]=[\mathrm{b}, \mathrm{r}, \mathrm{c}]} \\
& {[\mathrm{m}, \mathrm{s}]+[\mathrm{m}, \mathrm{b}]=[\mathrm{s}, \mathrm{m}, \mathrm{b}]} \\
& {[\mathrm{s}, \mathrm{v}]+[\mathrm{s}, \mathrm{c}]=[\mathrm{v}, \mathrm{s}, \mathrm{c}]} \\
& {[\mathrm{s}, \mathrm{v}]+[\mathrm{s}, \mathrm{m}]=[\mathrm{v}, \mathrm{s}, \mathrm{m}]} \\
& {[\mathrm{s}, \mathrm{v}]+[\mathrm{s}, \mathrm{r}]=[\mathrm{v}, \mathrm{s}, \mathrm{r}]} \\
& {[\mathrm{c}, \mathrm{m}]+[\mathrm{c}, \mathrm{b}]=[\mathrm{m}, \mathrm{c}, \mathrm{b}]} \\
& {[\mathrm{m}, \mathrm{r}]+[\mathrm{m}, \mathrm{c}]=[\mathrm{r}, \mathrm{m}, \mathrm{c}]} \\
& {[\mathrm{m}, \mathrm{b}]+[\mathrm{m}, \mathrm{c}]=[\mathrm{b}, \mathrm{m}, \mathrm{c}]} \\
& {[\mathrm{v}, \mathrm{b}]+[\mathrm{v}, \mathrm{m}]=[\mathrm{b}, \mathrm{v}, \mathrm{m}]} \\
& {[\mathrm{m}, \mathrm{r}]+[\mathrm{m}, \mathrm{v}]=[\mathrm{r}, \mathrm{m}, \mathrm{v}]} \\
& {[\mathrm{c}, \mathrm{r}]+[\mathrm{c}, \mathrm{s}]=[\mathrm{r}, \mathrm{c}, \mathrm{s}]} \\
& {[\mathrm{c}, \mathrm{v}]+[\mathrm{c}, \mathrm{b}]=[\mathrm{v}, \mathrm{c}, \mathrm{b}]} \\
& {[\mathrm{v}, \mathrm{b}]+[\mathrm{v}, \mathrm{c}]=[\mathrm{b}, \mathrm{v}, \mathrm{c}]} \\
& {[\mathrm{v}, \mathrm{b}]+[\mathrm{v}, \mathrm{r}]=[\mathrm{b}, \mathrm{v}, \mathrm{r}]} \\
& {[\mathrm{v}, \mathrm{m}]+[\mathrm{v}, \mathrm{s}]=[\mathrm{m}, \mathrm{v}, \mathrm{s}]} \\
& {[\mathrm{r}, \mathrm{s}]+[\mathrm{r}, \mathrm{c}]=[\mathrm{s}, \mathrm{r}, \mathrm{c}]} \\
& {[\mathrm{r}, \mathrm{v}]+[\mathrm{r}, \mathrm{m}]=[\mathrm{v}, \mathrm{r}, \mathrm{m}]}
\end{aligned}
$$

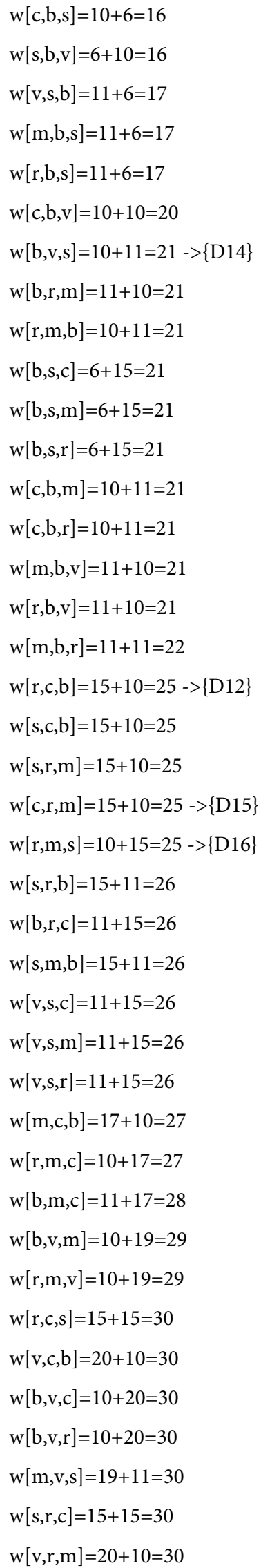

$$
\begin{array}{ll}
{[\mathrm{m}, \mathrm{v}]+[\mathrm{m}, \mathrm{b}]=[\mathrm{v}, \mathrm{m}, \mathrm{b}]} & \mathrm{w}[\mathrm{v}, \mathrm{m}, \mathrm{b}]=19+11=30 \\
{[\mathrm{~s}, \mathrm{c}]+[\mathrm{s}, \mathrm{m}]=[\mathrm{c}, \mathrm{s}, \mathrm{m}]} & \mathrm{w}[\mathrm{c}, \mathrm{s}, \mathrm{m}]=15+15=30 \\
{[\mathrm{~s}, \mathrm{c}]+[\mathrm{s}, \mathrm{r}]=[\mathrm{c}, \mathrm{s}, \mathrm{r}]} & \mathrm{w}[\mathrm{c}, \mathrm{s}, \mathrm{r}]=15+15=30 \\
{[\mathrm{~s}, \mathrm{~m}]+[\mathrm{s}, \mathrm{r}]=[\mathrm{m}, \mathrm{s}, \mathrm{r}]} & \mathrm{w}[\mathrm{m}, \mathrm{s}, \mathrm{r}]=15+15=30 \\
{[\mathrm{v}, \mathrm{c}]+[\mathrm{v}, \mathrm{s}]=[\mathrm{c}, \mathrm{v}, \mathrm{s}]} & \mathrm{w}[\mathrm{c}, \mathrm{v}, \mathrm{s}]=20+11=31 \\
{[\mathrm{v}, \mathrm{r}]+[\mathrm{v}, \mathrm{s}]=[\mathrm{r}, \mathrm{v}, \mathrm{s}]} & \mathrm{w}[\mathrm{r}, \mathrm{v}, \mathrm{s}]=20+11=31 \\
{[\mathrm{r}, \mathrm{v}]+[\mathrm{r}, \mathrm{b}]=[\mathrm{v}, \mathrm{r}, \mathrm{b}]} & \mathrm{w}[\mathrm{v}, \mathrm{r}, \mathrm{b}]=20+11=31 \\
{[\mathrm{c}, \mathrm{m}]+[\mathrm{c}, \mathrm{r}]=[\mathrm{m}, \mathrm{c}, \mathrm{r}]} & \mathrm{w}[\mathrm{m}, \mathrm{c}, \mathrm{r}]=17+15=32 \\
{[\mathrm{c}, \mathrm{m}]+[\mathrm{c}, \mathrm{s}]=[\mathrm{m}, \mathrm{c}, \mathrm{s}]} & \mathrm{w}[\mathrm{m}, \mathrm{c}, \mathrm{s}]=17+15=32 \\
{[\mathrm{~m}, \mathrm{~s}]+[\mathrm{m}, \mathrm{c}]=[\mathrm{s}, \mathrm{m}, \mathrm{c}]} & \mathrm{w}[\mathrm{s}, \mathrm{m}, \mathrm{c}]=15+17=32 \\
{[\mathrm{~m}, \mathrm{~s}]+[\mathrm{m}, \mathrm{v}]=[\mathrm{s}, \mathrm{m}, \mathrm{v}]} & \mathrm{w}[\mathrm{s}, \mathrm{m}, \mathrm{v}]=15+19=34 \\
{[\mathrm{c}, \mathrm{r}]+[\mathrm{c}, \mathrm{v}]=[\mathrm{r}, \mathrm{c}, \mathrm{v}]} & \mathrm{w}[\mathrm{r}, \mathrm{c}, \mathrm{v}]=15+20=35 \\
{[\mathrm{c}, \mathrm{s}]+[\mathrm{c}, \mathrm{v}]=[\mathrm{s}, \mathrm{c}, \mathrm{v}]} & \mathrm{w}[\mathrm{s}, \mathrm{c}, \mathrm{v}]=15+20=35 \\
{[\mathrm{r}, \mathrm{s}]+[\mathrm{r}, \mathrm{v}]=[\mathrm{s}, \mathrm{r}, \mathrm{v}]} & \mathrm{w}[\mathrm{s}, \mathrm{r}, \mathrm{v}]=15+20=35 \\
{[\mathrm{r}, \mathrm{v}]+[\mathrm{r}, \mathrm{c}]=[\mathrm{v}, \mathrm{r}, \mathrm{c}]} & \mathrm{w}[\mathrm{v}, \mathrm{r}, \mathrm{c}]=20+15=35 \\
{[\mathrm{~m}, \mathrm{v}]+[\mathrm{m}, \mathrm{c}]=[\mathrm{v}, \mathrm{m}, \mathrm{c}]} & \mathrm{w}[\mathrm{v}, \mathrm{m}, \mathrm{c}]=19+17=36 \\
{[\mathrm{c}, \mathrm{m}]+[\mathrm{c}, \mathrm{v}]=[\mathrm{m}, \mathrm{c}, \mathrm{v}]} & \mathrm{w}[\mathrm{m}, \mathrm{c}, \mathrm{v}]=17+20=37 \\
{[\mathrm{v}, \mathrm{c}]+[\mathrm{v}, \mathrm{m}]=[\mathrm{c}, \mathrm{v}, \mathrm{m}]} & \mathrm{w}[\mathrm{c}, \mathrm{v}, \mathrm{m}]=20+19=39 \\
{[\mathrm{v}, \mathrm{m}]+[\mathrm{v}, \mathrm{r}]=[\mathrm{m}, \mathrm{v}, \mathrm{r}]} & \mathrm{w}[\mathrm{m}, \mathrm{v}, \mathrm{r}]=19+20=39 \\
{[\mathrm{v}, \mathrm{c}]+[\mathrm{v}, \mathrm{r}]=[\mathrm{c}, \mathrm{v}, \mathrm{r}]} & \mathrm{w}[\mathrm{c}, \mathrm{v}, \mathrm{r}]=20+20=40
\end{array}
$$

->D12. [r,c,b] was used in forming the hamilton circuit.

->D14. $[\mathrm{b}, \mathrm{v}, \mathrm{s}]$ was used in forming the hamilton circuit.

->D15. [c,r,m] was used in forming the hamilton circuit.

$->\mathrm{D} 16$. $[\mathrm{r}, \mathrm{m}, \mathrm{s}]$ was used in forming the hamilton circuit.

$[\mathrm{c}, \mathrm{b}, \mathrm{s}],[\mathrm{s}, \mathrm{b}, \mathrm{v}],[\mathrm{v}, \mathrm{s}, \mathrm{b}],[\mathrm{m}, \mathrm{b}, \mathrm{s}],[\mathrm{r}, \mathrm{b}, \mathrm{s}],[\mathrm{c}, \mathrm{b}, \mathrm{v}],[\mathrm{b}, \mathrm{r}, \mathrm{m}],[\mathrm{r}, \mathrm{m}, \mathrm{b}],[\mathrm{b}, \mathrm{s}, \mathrm{c}]$, $[\mathrm{b}, \mathrm{s}, \mathrm{m}],[\mathrm{b}, \mathrm{s}, \mathrm{r}],[\mathrm{c}, \mathrm{b}, \mathrm{m}],[\mathrm{c}, \mathrm{b}, \mathrm{r}],[\mathrm{m}, \mathrm{b}, \mathrm{v}],[\mathrm{r}, \mathrm{b}, \mathrm{v}],[\mathrm{m}, \mathrm{b}, \mathrm{r}],[\mathrm{s}, \mathrm{c}, \mathrm{b}]$ and $[\mathrm{s}, \mathrm{r}, \mathrm{m}]$ was not used in forming the hamilton circuit even though they had lesser weights. So these partial circuits are taken individually and are used to form hamilton circuits ,to find whether these hamilton circuits have lesser weight than the hamilton circuit found out earlier (ie:- $[\mathrm{r}, \mathrm{c}, \mathrm{b}, \mathrm{v}, \mathrm{s}, \mathrm{m}, \mathrm{r}]$ was the hamilton circuit found out earlier). (The lesser weight partial circuits which were not used while forming the hamilton circuits for $[\mathrm{c}, \mathrm{b}, \mathrm{s}],[\mathrm{s}, \mathrm{b}, \mathrm{v}],[\mathrm{v}, \mathrm{s}, \mathrm{b}],[\mathrm{m}, \mathrm{b}, \mathrm{s}],[\mathrm{r}, \mathrm{b}, \mathrm{s}],[\mathrm{c}, \mathrm{b}, \mathrm{v}]$, $[\mathrm{b}, \mathrm{r}, \mathrm{m}],[\mathrm{r}, \mathrm{m}, \mathrm{b}],[\mathrm{b}, \mathrm{s}, \mathrm{c}],[\mathrm{b}, \mathrm{s}, \mathrm{m}],[\mathrm{b}, \mathrm{s}, \mathrm{r}],[\mathrm{c}, \mathrm{b}, \mathrm{m}],[\mathrm{c}, \mathrm{b}, \mathrm{r}],[\mathrm{m}, \mathrm{b}, \mathrm{v}],[\mathrm{r}, \mathrm{b}, \mathrm{v}]$, $[\mathrm{m}, \mathrm{b}, \mathrm{r}],[\mathrm{s}, \mathrm{c}, \mathrm{b}]$ and $[\mathrm{s}, \mathrm{r}, \mathrm{m}]$ need not be used for processing again).

Now first $[c, b, s]$ has to be taken as a necessary partial circuit and then the same process that occurred in Section \#2.7 has to be done.

1) So first $[c, b, s]$ is taken. Since hamilton circuit rule is not violated, $[\mathrm{c}, \mathrm{b}, \mathrm{s}]$ is taken.

2) Take $[r, c, b]$ from Vertex c. $[r, c, b]$ fits with $[c, b, s]$. So $[r, c, b]$ is taken. The new partial circuit is $[r, c, b, s]$.

3) Take $[b, v, s]$ from Vertex v. Here $[b, v, s]$ can replace $[b, s]$ of $[r, c, b, s]$, since according to the properties of joining of partial circuits, it is allowed. So $[\mathrm{b}, \mathrm{v}, \mathrm{s}]$ fits with $[\mathrm{r}, \mathrm{c}, \mathrm{b}, \mathrm{s}]$ to create the partial circuit $[\mathrm{r}, \mathrm{c}, \mathrm{b}, \mathrm{v}, \mathrm{s}]$.

4) Take $[b, r, m]$ from Vertex r. $[b, r, m]$ does not fit with $[r, c, b, v, s]$. 
So take [s,r,m] from Vertex r. [s,r,m] does not fit with [r,c,b,v,s]. So take $[\mathrm{c}, \mathrm{r}, \mathrm{m}]$ from Vertex $\mathrm{r}$. [c,r,m] fits with $[\mathrm{r}, \mathrm{c}, \mathrm{b}, \mathrm{v}, \mathrm{s}]$ to create the partial circuit $[\mathrm{m}, \mathrm{r}, \mathrm{c}, \mathrm{b}, \mathrm{v}, \mathrm{s}]$.

5) Take $[r, m, b]$ from Vertex $m$. [r,m,b] does not fit with [m,r,c,b,v,s]. So take [r,m,s] from Vertex m. [r,m,s] fits with [m,r,c,b,v,s] to create the hamilton circuit $[\mathrm{m}, \mathrm{r}, \mathrm{c}, \mathrm{b}, \mathrm{v}, \mathrm{s}, \mathrm{m}]$. Weight of the hamilton circuit $\mathrm{w}[\mathrm{m}, \mathrm{r}, \mathrm{c}, \mathrm{b}, \mathrm{v}, \mathrm{s}, \mathrm{m}]=\mathrm{w}[\mathrm{m}, \mathrm{r}]+\mathrm{w}[\mathrm{r}, \mathrm{c}]+\mathrm{w}[\mathrm{c}, \mathrm{b}]+\mathrm{w}[\mathrm{b}, \mathrm{v}]+\mathrm{w}[\mathrm{v}, \mathrm{s}]+$ $\mathrm{w}[\mathrm{s}, \mathrm{m}]=10+15+10+10+11+15=71$.

So $[\mathrm{m}, \mathrm{r}, \mathrm{c}, \mathrm{b}, \mathrm{v}, \mathrm{s}, \mathrm{m}]=[\mathrm{c}, \mathrm{b}, \mathrm{s}]+[\mathrm{r}, \mathrm{c}, \mathrm{b}]+[\mathrm{b}, \mathrm{v}, \mathrm{s}]+[\mathrm{c}, \mathrm{r}, \mathrm{m}]+[\mathrm{r}, \mathrm{m}, \mathrm{s}]$.

$\mathrm{w}[\mathrm{m}, \mathrm{r}, \mathrm{c}, \mathrm{b}, \mathrm{v}, \mathrm{s}, \mathrm{m}]=71$

Now $[\mathrm{s}, \mathrm{b}, \mathrm{v}]$ has to be taken as a necessary partial circuit and then the same process that occurred in Section \#2.7 has to be done.

1) First $[s, b, v]$ is taken. Since hamilton circuit rule is not violated, $[\mathrm{s}, \mathrm{b}, \mathrm{v}]$ is taken.

2) Take $[r, c, b]$ from Vertex $c$. $[r, c, b]$ does not fit with $[s, b, v]$. So take [s,c,b] from Vertex c. [s,c,b] can replace [s,b] of [s,b,v], since according to the properties of joining of partial circuits, it is allowed. So $[s, c, b]$ fits with $[\mathrm{s}, \mathrm{b}, \mathrm{v}]$ to form the partial circuit $[\mathrm{s}, \mathrm{c}, \mathrm{b}, \mathrm{v}]$.

3) Take $[b, v, s]$ from Vertex v. $[b, v, s]$ does not fit with $[s, c, b, v]$ because if $[\mathrm{b}, \mathrm{v}, \mathrm{s}]$ is joined with $[\mathrm{s}, \mathrm{c}, \mathrm{b}, \mathrm{v}]$, then it will form a hamilton ciruit $[\mathrm{s}, \mathrm{c}, \mathrm{b}, \mathrm{v}, \mathrm{s}]$ of 4 vertices, which is not what is required because a hamilton circuit of 6 vertices is required. So take $[\mathrm{b}, \mathrm{v}, \mathrm{m}]$ from vertex $\mathrm{v}$. $[\mathrm{b}, \mathrm{v}, \mathrm{m}]$ fits with $[\mathrm{s}, \mathrm{c}, \mathrm{b}, \mathrm{v}]$ to form the partial circuit $[\mathrm{s}, \mathrm{c}, \mathrm{b}, \mathrm{v}, \mathrm{m}]$.

4) Take $[b, r, m]$ from Vertex r. [b,r,m] does not fit with [s,c,b,v,m], since if $[\mathrm{b}, \mathrm{r}, \mathrm{m}]$ is made to join $[\mathrm{s}, \mathrm{c}, \mathrm{b}, \mathrm{v}, \mathrm{m}]$, then $[\mathrm{b}, \mathrm{r}, \mathrm{m}]$ will replace $[\mathrm{b}, \mathrm{v}, \mathrm{m}]$ of $[\mathrm{s}, \mathrm{c}, \mathrm{b}, \mathrm{v}, \mathrm{m}]$, so in the process if vertex $\mathrm{r}$ is gained, then vertex $\mathrm{v}$ is lost. So such joining is not allowed according to the principle of joining of partial circuits given in the top section of the article. Take $[\mathrm{s}, \mathrm{r}, \mathrm{m}]$ of vertex r. [s,r,m] fits with $[\mathrm{s}, \mathrm{c}, \mathrm{b}, \mathrm{v}, \mathrm{m}]$

to form the hamilton circuit $[\mathrm{s}, \mathrm{c}, \mathrm{b}, \mathrm{v}, \mathrm{m}, \mathrm{r}, \mathrm{s}]$. The weight of the hamilton circuit $w[s, c, b, v, m, r, s]=w[s, c]+w[c, b]+w[b, v]+w[v, m]+$ $\mathrm{w}[\mathrm{m}, \mathrm{r}]+\mathrm{w}[\mathrm{r}, \mathrm{s}]=79$

$$
\begin{aligned}
& \text { So }[\mathrm{s}, \mathrm{c}, \mathrm{b}, \mathrm{v}, \mathrm{m}, \mathrm{r}, \mathrm{s}]=[\mathrm{s}, \mathrm{b}, \mathrm{v}]+[\mathrm{s}, \mathrm{c}, \mathrm{b}]+[\mathrm{b}, \mathrm{v}, \mathrm{m}]+[\mathrm{s}, \mathrm{r}, \mathrm{m}] \\
& \mathrm{w}[\mathrm{s}, \mathrm{c}, \mathrm{b}, \mathrm{v}, \mathrm{m}, \mathrm{r}, \mathrm{s}]=79
\end{aligned}
$$

Now $[\mathrm{v}, \mathrm{s}, \mathrm{b}]$ has to be taken as a necessary partial circuit and then the same process that occurred in Section \#2.7 has to be done.

1) First $[v, s, b]$ is taken. Since hamilton circuit rule is not violated, $[\mathrm{v}, \mathrm{s}, \mathrm{b}]$ is taken.

2) Take $[r, c, b]$ from Vertex c. $[r, c, b]$ fits with $[v, s, b]$ to form the partial circuit $[\mathrm{v}, \mathrm{s}, \mathrm{b}, \mathrm{c}, \mathrm{r}]$

3) Take $[b, v, s]$ from vertex v. $[b, v, s]$ does not fit with $[v, s, b, c, r]$. Because here $[b, s]$ of $[v, s, b, c, r]$ may replace $[b, v]$ of $[b, v, s]$ but the weight of [b,v] (ie:- 10) is more than the weight of [b,s] (ie:- 6) being replaced. Also the edge may be replaced, because no vertices are lost and an edge is replaced by another edge. But the weight here is greater, so it is not replaced. Take $[\mathrm{b}, \mathrm{v}, \mathrm{m}]$ from Vertex $\mathrm{v}$. [b,v,m] does not fit with $[\mathrm{v}, \mathrm{s}, \mathrm{b}, \mathrm{c}, \mathrm{r}]$. So also $[\mathrm{b}, \mathrm{v}, \mathrm{c}]$ and $[\mathrm{b}, \mathrm{v}, \mathrm{r}]$ of vertex $\mathrm{v}$ does not fit with $[\mathrm{v}, \mathrm{s}, \mathrm{b}, \mathrm{c}, \mathrm{r}]$. Take $[\mathrm{m}, \mathrm{v}, \mathrm{s}]$ of vertex $\mathrm{v}$. $[\mathrm{m}, \mathrm{v}, \mathrm{s}]$ fits with $[\mathrm{v}, \mathrm{s}, \mathrm{b}, \mathrm{c}, \mathrm{r}]$ to form the partial circuit $[\mathrm{m}, \mathrm{v}, \mathrm{s}, \mathrm{b}, \mathrm{c}, \mathrm{r}]$.

4) Take $[b, r, m]$ from Vertex r. [b,r,m] does not fit with $[\mathrm{m}, \mathrm{v}, \mathrm{s}, \mathrm{b}, \mathrm{c}, \mathrm{r}]$. Take $[\mathrm{s}, \mathrm{r}, \mathrm{m}]$ from Vertex r. [s,r,m] does not fit with [m,v,s,b,c,r]. Take $[\mathrm{c}, \mathrm{r}, \mathrm{m}]$ from Vertex $\mathrm{r}$. [c,r,m] fits with $[\mathrm{m}, \mathrm{v}, \mathrm{s}, \mathrm{b}, \mathrm{c}, \mathrm{r}]$ to create the hamilton circuit $[\mathrm{m}, \mathrm{v}, \mathrm{s}, \mathrm{b}, \mathrm{c}, \mathrm{r}, \mathrm{m}]$. The weight of the hamilton circuit $\mathrm{w}[\mathrm{m}, \mathrm{v}, \mathrm{s}, \mathrm{b}, \mathrm{c}, \mathrm{r}, \mathrm{m}]=\mathrm{w}[\mathrm{m}, \mathrm{v}]+\mathrm{w}[\mathrm{v}, \mathrm{s}]+\mathrm{w}[\mathrm{s}, \mathrm{b}]+\mathrm{w}[\mathrm{b}, \mathrm{c}]+\mathrm{w}[\mathrm{c}, \mathrm{r}]+$ $\mathrm{w}[\mathrm{r}, \mathrm{m}]=71$.

$$
\begin{aligned}
& \text { So }[\mathrm{m}, \mathrm{v}, \mathrm{s}, \mathrm{b}, \mathrm{c}, \mathrm{r}, \mathrm{m}]=[\mathrm{v}, \mathrm{s}, \mathrm{b}]+[\mathrm{r}, \mathrm{c}, \mathrm{b}]+[\mathrm{m}, \mathrm{v}, \mathrm{s}]+[\mathrm{c}, \mathrm{r}, \mathrm{m}] \\
& \mathrm{w}[\mathrm{m}, \mathrm{v}, \mathrm{s}, \mathrm{b}, \mathrm{c}, \mathrm{r}, \mathrm{m}]=71
\end{aligned}
$$

In the same way that the hamilton circuit has been obtained for $[\mathrm{c}, \mathrm{b}, \mathrm{s}],[\mathrm{s}, \mathrm{b}, \mathrm{v}]$ and $[\mathrm{v}, \mathrm{s}, \mathrm{b}]$, it is also done here for [m,b,s], [r,b,s], [c,b,v], $[\mathrm{b}, \mathrm{r}, \mathrm{m}],[\mathrm{r}, \mathrm{m}, \mathrm{b}],[\mathrm{b}, \mathrm{s}, \mathrm{c}],[\mathrm{b}, \mathrm{s}, \mathrm{m}],[\mathrm{b}, \mathrm{s}, \mathrm{r}],[\mathrm{c}, \mathrm{b}, \mathrm{m}],[\mathrm{c}, \mathrm{b}, \mathrm{r}],[\mathrm{m}, \mathrm{b}, \mathrm{v}],[\mathrm{r}, \mathrm{b}, \mathrm{v}]$, $[\mathrm{m}, \mathrm{b}, \mathrm{r}],[\mathrm{s}, \mathrm{c}, \mathrm{b}]$ and $[\mathrm{s}, \mathrm{r}, \mathrm{m}]$. Only the final result is written below in order to make the example as brief as possible.

Taking $[\mathrm{m}, \mathrm{b}, \mathrm{s}]$ as a necessay partial circuit, the hamilton circuit for $[\mathrm{m}, \mathrm{b}, \mathrm{s}]$ is :-

$$
\begin{aligned}
& {[\mathrm{m}, \mathrm{v}, \mathrm{b}, \mathrm{c}, \mathrm{s}, \mathrm{r}, \mathrm{m}]=[\mathrm{m}, \mathrm{b}, \mathrm{s}]+[\mathrm{s}, \mathrm{c}, \mathrm{b}]+[\mathrm{b}, \mathrm{v}, \mathrm{m}]+[\mathrm{s}, \mathrm{r}, \mathrm{m}]} \\
& \mathrm{w}[\mathrm{m}, \mathrm{v}, \mathrm{b}, \mathrm{c}, \mathrm{s}, \mathrm{r}, \mathrm{m}]=79
\end{aligned}
$$

Taking $[r, b, s]$ as a necessary partial circuit, the hamilton circuit for $[\mathrm{r}, \mathrm{b}, \mathrm{s}]$ is :-

$$
\begin{aligned}
& {[\mathrm{r}, \mathrm{m}, \mathrm{s}, \mathrm{v}, \mathrm{b}, \mathrm{c}, \mathrm{r}]=[\mathrm{r}, \mathrm{b}, \mathrm{s}]+[\mathrm{r}, \mathrm{c}, \mathrm{b}]+[\mathrm{b}, \mathrm{v}, \mathrm{s}]+[\mathrm{c}, \mathrm{r}, \mathrm{m}]+[\mathrm{r}, \mathrm{m}, \mathrm{s}]} \\
& \mathrm{w}[\mathrm{r}, \mathrm{m}, \mathrm{s}, \mathrm{v}, \mathrm{b}, \mathrm{c}, \mathrm{r}]=78
\end{aligned}
$$

Taking $[\mathrm{c}, \mathrm{b}, \mathrm{v}]$ as a necessary partial circuit, the hamilton circuit for $[c, b, v]$ is :-

$$
[\mathrm{c}, \mathrm{b}, \mathrm{v}, \mathrm{s}, \mathrm{m}, \mathrm{r}, \mathrm{c}]=[\mathrm{c}, \mathrm{b}, \mathrm{v}]+[\mathrm{r}, \mathrm{c}, \mathrm{b}]+[\mathrm{b}, \mathrm{v}, \mathrm{s}]+[\mathrm{c}, \mathrm{r}, \mathrm{m}]+[\mathrm{r}, \mathrm{m}, \mathrm{s}]
$$$$
\mathrm{w}[\mathrm{c}, \mathrm{b}, \mathrm{v}, \mathrm{s}, \mathrm{m}, \mathrm{r}, \mathrm{c}]=71
$$

Taking $[\mathrm{b}, \mathrm{r}, \mathrm{m}]$ as a necessary partial circuit, the hamilton circuit for $[\mathrm{b}, \mathrm{r}, \mathrm{m}]$ is:-

$[\mathrm{b}, \mathrm{c}, \mathrm{r}, \mathrm{m}, \mathrm{s}, \mathrm{v}, \mathrm{b}]=[\mathrm{b}, \mathrm{r}, \mathrm{m}]+[\mathrm{r}, \mathrm{c}, \mathrm{b}]+[\mathrm{b}, \mathrm{v}, \mathrm{s}]+[\mathrm{r}, \mathrm{m}, \mathrm{s}]$.

$\mathrm{w}[\mathrm{b}, \mathrm{c}, \mathrm{r}, \mathrm{m}, \mathrm{s}, \mathrm{v}, \mathrm{b}]=71$

Taking $[\mathrm{r}, \mathrm{m}, \mathrm{b}]$ as a necessary partial circuit, the hamilton circuit for $[\mathrm{r}, \mathrm{m}, \mathrm{b}]$ is:-

$$
\begin{aligned}
& {[\mathrm{r}, \mathrm{m}, \mathrm{v}, \mathrm{b}, \mathrm{c}, \mathrm{s}, \mathrm{r}]=[\mathrm{r}, \mathrm{m}, \mathrm{b}]+[\mathrm{s}, \mathrm{c}, \mathrm{b}]+[\mathrm{b}, \mathrm{v}, \mathrm{m}]+[\mathrm{s}, \mathrm{r}, \mathrm{m}]} \\
& \mathrm{w}[\mathrm{r}, \mathrm{m}, \mathrm{v}, \mathrm{b}, \mathrm{c}, \mathrm{s}, \mathrm{r}]=79
\end{aligned}
$$

Taking $[\mathrm{b}, \mathrm{s}, \mathrm{c}]$ as a necessary partial circuit, the hamilton circuit for $[\mathrm{b}, \mathrm{s}, \mathrm{c}]$ is:-

$$
\begin{aligned}
& {[\mathrm{b}, \mathrm{v}, \mathrm{s}, \mathrm{c}, \mathrm{r}, \mathrm{m}, \mathrm{b}]=[\mathrm{b}, \mathrm{s}, \mathrm{c}]+[\mathrm{r}, \mathrm{c}, \mathrm{s}]+[\mathrm{b}, \mathrm{v}, \mathrm{s}]+[\mathrm{c}, \mathrm{r}, \mathrm{m}]+[\mathrm{r}, \mathrm{m}, \mathrm{b}]} \\
& \mathrm{w}[\mathrm{b}, \mathrm{v}, \mathrm{s}, \mathrm{c}, \mathrm{r}, \mathrm{m}, \mathrm{b}]=72
\end{aligned}
$$

Taking $[\mathrm{b}, \mathrm{s}, \mathrm{m}]$ as a necessary partial circuit, the hamilton circuit for $[b, s, m]$ is:-

$$
\begin{aligned}
& {[\mathrm{b}, \mathrm{v}, \mathrm{s}, \mathrm{m}, \mathrm{r}, \mathrm{c}, \mathrm{b}]=[\mathrm{b}, \mathrm{s}, \mathrm{m}]+[\mathrm{r}, \mathrm{c}, \mathrm{b}]+[\mathrm{b}, \mathrm{v}, \mathrm{s}]+[\mathrm{c}, \mathrm{r}, \mathrm{m}]} \\
& \mathrm{w}[\mathrm{b}, \mathrm{v}, \mathrm{s}, \mathrm{m}, \mathrm{r}, \mathrm{c}, \mathrm{b}]=71
\end{aligned}
$$

Taking $[\mathrm{b}, \mathrm{s}, \mathrm{r}]$ as a necessary partial circuit, the hamilton circuit for $[\mathrm{b}, \mathrm{s}, \mathrm{r}]$ is:-

$$
\begin{aligned}
& {[\mathrm{b}, \mathrm{c}, \mathrm{s}, \mathrm{r}, \mathrm{m}, \mathrm{v}, \mathrm{b}]=[\mathrm{b}, \mathrm{s}, \mathrm{r}]+[\mathrm{s}, \mathrm{c}, \mathrm{b}]+[\mathrm{b}, \mathrm{v}, \mathrm{m}]+[\mathrm{s}, \mathrm{r}, \mathrm{m}]} \\
& \mathrm{w}[\mathrm{b}, \mathrm{c}, \mathrm{s}, \mathrm{r}, \mathrm{m}, \mathrm{v}, \mathrm{b}]=79
\end{aligned}
$$

Taking $[\mathrm{c}, \mathrm{b}, \mathrm{m}]$ as a necessary partial circuit, the hamilton circuit for $[\mathrm{c}, \mathrm{b}, \mathrm{m}]$ is:-

$[\mathrm{c}, \mathrm{b}, \mathrm{v}, \mathrm{m}, \mathrm{s}, \mathrm{r}, \mathrm{c}]=[\mathrm{c}, \mathrm{b}, \mathrm{m}]+[\mathrm{r}, \mathrm{c}, \mathrm{b}]+[\mathrm{b}, \mathrm{v}, \mathrm{m}]+[\mathrm{s}, \mathrm{r}, \mathrm{c}]+[\mathrm{s}, \mathrm{m}, \mathrm{v}]$ 
$\mathrm{w}[\mathrm{c}, \mathrm{b}, \mathrm{v}, \mathrm{m}, \mathrm{s}, \mathrm{r}, \mathrm{c}]=84$

Taking $[c, b, r]$ as a necessary partial circuit, the hamilton circuit for $[c, b, r]$ is:-

$[\mathrm{c}, \mathrm{v}, \mathrm{b}, \mathrm{r}, \mathrm{m}, \mathrm{s}, \mathrm{c}]=[\mathrm{c}, \mathrm{b}, \mathrm{r}]+[\mathrm{s}, \mathrm{c}, \mathrm{b}]+[\mathrm{b}, \mathrm{v}, \mathrm{c}]+[\mathrm{b}, \mathrm{r}, \mathrm{m}]+[\mathrm{r}, \mathrm{m}, \mathrm{s}]$

$\mathrm{w}[\mathrm{c}, \mathrm{v}, \mathrm{b}, \mathrm{r}, \mathrm{m}, \mathrm{s}, \mathrm{c}]=81$

Taking $[\mathrm{m}, \mathrm{b}, \mathrm{v}]$ as a necessary partial circuit, the hamilton circuit for $[\mathrm{m}, \mathrm{b}, \mathrm{v}]$ is:-

$[\mathrm{m}, \mathrm{c}, \mathrm{b}, \mathrm{v}, \mathrm{s}, \mathrm{r}, \mathrm{m}]=[\mathrm{m}, \mathrm{b}, \mathrm{v}]+[\mathrm{m}, \mathrm{c}, \mathrm{b}]+[\mathrm{b}, \mathrm{v}, \mathrm{s}]+[\mathrm{s}, \mathrm{r}, \mathrm{m}]$

$\mathrm{w}[\mathrm{m}, \mathrm{c}, \mathrm{b}, \mathrm{v}, \mathrm{s}, \mathrm{r}, \mathrm{m}]=73$

Taking $[\mathrm{r}, \mathrm{b}, \mathrm{v}]$ as a necessary partial circuit, the hamilton circuit for $[\mathrm{r}, \mathrm{b}, \mathrm{v}]$ is:-

$[\mathrm{r}, \mathrm{m}, \mathrm{s}, \mathrm{v}, \mathrm{b}, \mathrm{c}, \mathrm{r}]=[\mathrm{r}, \mathrm{b}, \mathrm{v}]+[\mathrm{r}, \mathrm{c}, \mathrm{b}]+[\mathrm{b}, \mathrm{v}, \mathrm{s}]+[\mathrm{c}, \mathrm{r}, \mathrm{m}]$

$\mathrm{w}[\mathrm{r}, \mathrm{m}, \mathrm{s}, \mathrm{v}, \mathrm{b}, \mathrm{c}, \mathrm{r}]=71$

Taking $[\mathrm{m}, \mathrm{b}, \mathrm{r}]$ as a necessary partial circuit, the hamilton circuit for $[\mathrm{m}, \mathrm{b}, \mathrm{r}]$ is:-

$$
\begin{aligned}
& {[\mathrm{m}, \mathrm{s}, \mathrm{r}, \mathrm{c}, \mathrm{b}, \mathrm{v}, \mathrm{m}]=[\mathrm{m}, \mathrm{b}, \mathrm{r}]+[\mathrm{r}, \mathrm{c}, \mathrm{b}]+[\mathrm{b}, \mathrm{v}, \mathrm{m}]+[\mathrm{s}, \mathrm{r}, \mathrm{c}]+[\mathrm{s}, \mathrm{m}, \mathrm{v}]} \\
& \mathrm{w}[\mathrm{m}, \mathrm{s}, \mathrm{r}, \mathrm{c}, \mathrm{b}, \mathrm{v}, \mathrm{m}]=84
\end{aligned}
$$

Taking $[\mathrm{s}, \mathrm{c}, \mathrm{b}]$ as a necessary partial circuit, the hamilton circuit for $[\mathrm{s}, \mathrm{c}, \mathrm{b}]$ is:-

$$
\begin{aligned}
& {[\mathrm{s}, \mathrm{c}, \mathrm{b}, \mathrm{v}, \mathrm{m}, \mathrm{r}, \mathrm{s}]=[\mathrm{s}, \mathrm{c}, \mathrm{b}]+[\mathrm{b}, \mathrm{v}, \mathrm{m}]+[\mathrm{s}, \mathrm{r}, \mathrm{m}]} \\
& \mathrm{w}[\mathrm{s}, \mathrm{c}, \mathrm{b}, \mathrm{v}, \mathrm{m}, \mathrm{r}, \mathrm{s}]=79
\end{aligned}
$$

Taking $[\mathrm{s}, \mathrm{r}, \mathrm{m}]$ as a necessary partial circuit, the hamilton circuit for $[\mathrm{s}, \mathrm{r}, \mathrm{m}]$ is:-

$$
\begin{aligned}
& {[\mathrm{s}, \mathrm{r}, \mathrm{m}, \mathrm{v}, \mathrm{b}, \mathrm{c}, \mathrm{s}]=[\mathrm{s}, \mathrm{r}, \mathrm{m}]+[\mathrm{s}, \mathrm{c}, \mathrm{b}]+[\mathrm{b}, \mathrm{v}, \mathrm{m}]} \\
& \mathrm{w}[\mathrm{s}, \mathrm{r}, \mathrm{m}, \mathrm{v}, \mathrm{b}, \mathrm{c}, \mathrm{s}]=79
\end{aligned}
$$

The lowest weight for a hamilton circuit is 71 among the hamilton circuits taken for the partial circuits of $[\mathrm{c}, \mathrm{b}, \mathrm{s}],[\mathrm{s}, \mathrm{b}, \mathrm{v}],[\mathrm{v}, \mathrm{s}, \mathrm{b}],[\mathrm{m}, \mathrm{b}, \mathrm{s}]$, $[\mathrm{r}, \mathrm{b}, \mathrm{s}],[\mathrm{c}, \mathrm{b}, \mathrm{v}],[\mathrm{b}, \mathrm{r}, \mathrm{m}],[\mathrm{r}, \mathrm{m}, \mathrm{b}],[\mathrm{b}, \mathrm{s}, \mathrm{c}],[\mathrm{b}, \mathrm{s}, \mathrm{m}],[\mathrm{b}, \mathrm{s}, \mathrm{r}],[\mathrm{c}, \mathrm{b}, \mathrm{m}],[\mathrm{c}, \mathrm{b}, \mathrm{r}]$, $[\mathrm{m}, \mathrm{b}, \mathrm{v}],[\mathrm{r}, \mathrm{b}, \mathrm{v}],[\mathrm{m}, \mathrm{b}, \mathrm{r}],[\mathrm{s}, \mathrm{c}, \mathrm{b}]$ and $[\mathrm{s}, \mathrm{r}, \mathrm{m}]$. Also the weight of the Hamilton circuit obtained in Section \#2.7 is also 71. So the Hamilton circuit obtained in Section \#2.7 or in Section \#2.9 with weight of "71" is taken as the minimum weight Hamilton circuit.

Section \#2.9 (END).

\section{References}

1. Liu CL (1985) Elements of Discrete Mathematics. (2nd Edition), McGraw-Hill Inc., US 433.

2. Clark J, Holton DA (1991) A First Look at Graph Theory. World Scientific Pub Co Inc 330. 